\title{
The Weak Null Condition in Free-evolution Schemes for Numerical Relativity: Dual Foliation GHG with Constraint Damping
}

\author{
Edgar Gasperín and David Hilditch \\ Centro de Astrofísica e Gravitação - CENTRA, Departamento de Física, Instituto Superior Técnico - IST, \\ Universidade de Lisboa - UL, Av. Rovisco Pais 1, 1049-001 Lisboa, Portugal
}

(Dated: December 18, 2018)

\begin{abstract}
All strategies for the treatment of future null-infinity in numerical relativity involve some form of regularization of the field equations. In a recent proposal that relies on the dual foliation formalism this is achieved by the use of an asymptotically Minkowskian generalized harmonic tensor basis. For the scheme to work however, derivatives of certain coordinate light-speeds must decay fast enough. Presently, we generalize the method of asymptotic expansions for nonlinear wave equations to treat first order symmetric hyperbolic systems. We then use this heuristic tool to extract the expected rates of decay of the metric near null-infinity in a free-evolution setting. We show, within the asymptotic expansion, that by carefully modifying the non-principal part of the field equations by the addition of constraints, we are able to obtain optimal decay rates even when the constraints are violated. The light-speed condition can hence be satisfied, which paves the way for the explicit numerical treatment of future null-infinity. We then study the behavior of the Trautman-Bondi mass under the decay results predicted by the asymptotic expansion. Naively the mass seems to be unbounded, but we see first that the divergent terms can be replaced with a combination of the constraints and the Einstein field equations, and second that the Bondi mass loss formula is recovered within the framework. Both of the latter results hold in the presence of small constraint violations.
\end{abstract}

Contents

\section{Introduction}

II. Basic set up

III. The asymptotic system for first and second order systems

A. Second order systems

B. First order systems

IV. A constraint damped hyperbolic reduction of GR in second order form

A. Hyperbolic reductions of GR

B. The asymptotic system and the weak null condition

C. The coordinate light-speed condition

V. The asymptotic system for $\mathcal{T}_{a b}={ }^{\mathscr{I}} \mathcal{T}_{a b}$

A. Analysis of the asymptotic system

B. The eikonal equation

VI. The asymptotic system for first order GHG with constraint damping

A. First order GHG

B. Evolution equations for the perturbation

VII. The Trautman-Bondi mass

VIII. Conclusions

Acknowledgments

References

\section{INTRODUCTION}

1

2

3

田
The notion of gravitational radiation is intimately related to the asymptotic behavior of the gravitational field. According to Penrose's proposal [1-3] isolated systems may be described by asymptotically simple spacetimes. Among these spacetimes those with vanishing cosmological constant, also known as Minkowski-like spacetimes, play a fundamental role for the current understanding of gravitational radiation [4-7]. For these spacetimes, the conformal boundary $\mathscr{I}$ is a null-hypersurface representing idealized observers at infinity. It is in this setting, in which the concept of gravitational radiation, originally introduced at the linear level, can be made rigorous in the non-linear theory and results such as the loss of mass-energy due to outgoing gravitational radiation [8 10] properly formulated.

Despite the advances achieved in numerical relativity (NR) in the last two decades, the inclusion of future null infinity in the computational domain is in general still an open problem. In view of the first direct detection of gravitational waves in 2016 [11] and the further development of gravitational wave astronomy in the years to come, the most natural motivation to solve this problem is for the computation of astrophysical wave forms at future null infinity. Nevertheless there are other important principle reasons to include the conformal boundary in the numerical domain, not least the weak cosmic censorship conjecture.

There are several approaches to include the conformal boundary in the computational domain. Possible avenues include Cauchy-Characteristic Matching [12] and the use of a suitable hyperboloidal initial value problem. In the latter approach, which we follow, initial data is given 
on slices which are everywhere spacelike but which terminate at future null infinity. This is combined with a suitable radial compactification. Naively looking at the field equations on such a slice however we get singular expressions, for which some cure is needed, particularly for numerical applications. One option is to use the conformal Einstein field equations introduced by Friedrich in 13. Although this approach is well suited to study the asymptotic region of the spacetime, it poses difficulties to numerically evolve realistic astrophysical scenarios, as it is not clear how to adapt those codes and techniques that already work well in the strong field region, for instance for compact binaries. Recent progress on the numerical implementation of the conformal Einstein field equations has however been reported in 14. Another proposal is to use a standard formulation of GR in generalized harmonic gauge (GHG) but insist on performing a full compactification of spacetime. This leads to equations which are formally singular but which take a finite limit in a suitable gauge [15. Following up on these ideas, Vañó-Viñuales and collaborators have shown successful numerical evolutions in spherical symmetry [16 18]. See also [19, 20] for other related approaches.

An alternative proposal, using the coordinates of [21], was given in 22 . Here the strategy, broadly speaking, is to pose an hyperboloidal initial value problem and exploit the dual foliation formalism 23 . In the dual foliation formalism two coordinate systems with two different foliations are employed. This allows us to use one coordinate system to construct a global tensor basis and another to coordinatize the spacetime. In the current application, it is natural to take the first coordinate system adapted to a Cauchy hypersurface, and the other to a hyperboloidal slice [22]. In this proposal, one employs a first order reduction of GHG. The regularization strategy is based on the idea that, near null infinity, the metric looks trivial in the GHG tensor basis. It was found in 22 that for the regularization to work certain derivatives of the coordinate light-speeds must have enough decay. We call this the coordinate light-speed condition.

In this article we analyze whether or not this condition can be realized and if so, in which circumstances. Interestingly, this is connected with the the weak null condition [24] and, consequently, to the concept of asymptotic systems. The asymptotic system can be regarded as a heuristic tool to predict the asymptotics of the solutions to a general a system of quasilinear wave equations. We view this tool as heuristic because, to the best of our knowledge, it has not yet been established that solutions to the asymptotic system will always have the same asymptotics as solutions to the original system.

It has however been shown that if a system of wave equations satisfy the hierarchical weak null condition, a condition slightly stronger than the weak null condition, then, the original PDEs admit global solutions whose asymptotics agree with the prediction of the asymptotic system [25]. An important consequence is that global existence for GR in harmonic gauge can be estab- lished without assuming that the constraints are satisfied. Moreover when the constraints are satisfied the formulation of 24 is expected to satisfy the coordinate lightspeed condition, so naively the situation looks promising. In free evolution schemes however, constraint violations are not only present but expected to grow rapidly, rendering the solution unphysical $26-28$. Therefore the use of the field equations as in 24 is not appropriate for numerical free evolution as envisioned in our program. This is an old problem in NR, and various approaches have been suggested to alleviate it. For instance in [27] a constraint addition was proposed which effectively damps away high frequency violations at the linear level. Unfortunately for our desired application the formulation of [24, 25] is not expected to satisfy the coordinate light-speed condition in the presence constraint violations. Presently we solve the problem with a GHG formulation, making a careful constraint addition to the field equations. With this addition the corresponding asymptotic system predicts that constraint violations are strongly damped as one approaches null infinity, and we recover the same fall-off present in their absence. This in turn should ensure that the coordinate light-speed condition of 22 is satisfied even in the presence of small constraint violations.

Although the calculation of the asymptotic system is straightforward, most of the literature about asymptotic systems [24, 29, 30] has been given in the context of second order equations. In view of the fact that the formulation of the Einstein field equations used in 22 is first order, we give a discussion of how to compute the asymptotic system for such systems. Finally we discuss the connection between the Trautman-Bondi mass and the asymptotic system and recover the mass loss formula in this context. We also see that modifying the definition of the Trautman-Bondi mass with the constraints, similar results may be obtained even when small constraint violations are present.

\section{BASIC SET UP}

In what follows, lower case Latin letters from the first half of the alphabet will denote abstract indices while Greek letters will be used to denote coordinate indices. In addition, capital Latin letters will be use to label the elements of a non-coordinate frame basis (the angular part of a spacetime null frame). Given a 2-tensor $T_{a b}$ and arbitrary frame vector fields $X^{a}, Y^{b}$ the components of $T_{a b}$ in this frame will be denoted as $T_{X X}=X^{a} X^{b} T_{a b}$, $T_{X Y}=X^{a} Y^{b} T_{a b}, T_{Y X}=Y^{a} X^{b} T_{a b}$ and $T_{Y Y}=Y^{a} Y^{b} T_{a b}$ and similarly for higher-valence tensors. Let $m_{a b}$ and $\nabla^{\circ}$ represent the Minkowski metric and its corresponding Levi-Civita connection. Let $\left(T, R, \theta^{A}\right)$ denote spherical polar coordinates where $\theta^{A}$ with $A \in\{1,2\}$ represent some arbitrary coordinates on $\mathbb{S}^{2}$. Let $\omega_{A}{ }^{a}$ with $A \in$ $\{1,2\}$ denote a frame with corresponding coframe $\hat{\omega}^{A}{ }_{a}$, 
such that

$$
\sigma_{a b}=\delta_{A B} \hat{\omega}^{A}{ }_{a} \hat{\omega}^{B}{ }_{b}
$$

where $\sigma_{a b}$ represents the standard metric on $\mathbb{S}^{2}$ and $\delta_{A B}$ is the Kronecker delta. Observe that, $\omega_{A}{ }^{a}$ is not a coordinate frame. In fact, respect to the natural coordinate basis one can write

$$
\hat{\omega}_{a}^{A}=\Omega_{B}^{A}\left(\mathrm{~d} \theta^{B}\right)_{a},
$$

where $\Omega_{B}{ }^{A}$ is a matrix which only depends on the coordinates $\theta^{A}$. In this frame, the Minkowski metric can be expressed in abstract index notation as

$$
m_{a b}=-(\mathrm{d} T)_{a}(\mathrm{~d} T)_{b}+(\mathrm{d} R)_{a}(\mathrm{~d} R)_{b}+R^{2} \sigma_{a b} .
$$

Let $\left(L^{a}, \underline{L}^{a}, S_{A}{ }^{a}\right)$ denote a null frame respect to $m_{a b}$ with the following normalization

$$
\begin{gathered}
m_{L \underline{L}}=-2, \quad m_{S_{A} S_{B}}=\delta_{A B}, \\
m_{L L}=m_{\underline{L L}}=m_{L S_{A}}=m_{\underline{L} S_{A}}=0 .
\end{gathered}
$$

The relation between the flat null frame $\left(L^{a}, \underline{L}^{a}, S_{A}{ }^{a}\right)$ and $\left(\left(\partial_{T}\right)^{a},\left(\partial_{R}\right)^{a}, \omega_{A}^{a}\right)$ is given by

$$
\begin{aligned}
L^{a} & =\left(\partial_{T}\right)^{a}+\left(\partial_{R}\right)^{a}, \quad \underline{L}^{a}=\left(\partial_{T}\right)^{a}-\left(\partial_{R}\right)^{a}, \\
S_{A}{ }^{a} & =\frac{1}{R} \omega_{A}{ }^{a} .
\end{aligned}
$$

Similarly, let $\left(\hat{L}_{a}, \hat{\underline{L}}_{a}, \hat{S}^{A}{ }_{a}\right)$ denote the associated coframe. Observe that in terms of the corresponding coframe $\left((\mathrm{d} T)_{a},(\mathrm{~d} R)_{a}, \omega^{A}{ }_{a}\right)$ one has

$$
\begin{aligned}
\hat{L}_{a} & =-(\mathrm{d} T)_{a}+(\mathrm{d} R)_{a}, \quad \hat{L}_{a}=-(\mathrm{d} T)_{a}-(\mathrm{d} R)_{a}, \\
\hat{S}_{a}^{A} & =R \hat{\omega}^{A}{ }_{a} .
\end{aligned}
$$

Therefore, the Minkowski metric can be succinctly expressed as

$$
m_{a b}=-\hat{L}_{(a} \underline{\hat{L}}_{b)}+\delta_{A B} \hat{S}^{A}{ }_{a} \hat{S}^{B}{ }_{b} .
$$

More generally, observe that, given a symmetric tensor $T_{a b}=T_{(a b)}$ one can express it as,

$$
\begin{aligned}
T_{a b} & =\frac{1}{4} T_{\underline{L L}} \hat{L}_{a} \hat{L}_{b}+\frac{1}{2} T_{L \underline{L}} \hat{L}_{(a} \hat{\underline{L}}_{b)}+\frac{1}{4} T_{L L} \underline{\hat{L}}_{a} \underline{\hat{L}}_{b}+T_{A B}^{\mathrm{TF}} \perp_{a b}^{A B} \\
& +\frac{1}{2} T^{\varnothing} \delta_{A B} \hat{S}^{A}{ }_{a} \hat{S}^{B}{ }_{b}-T_{\underline{L}_{A}} \hat{L}_{(a} \hat{S}^{A}{ }_{b)}-T_{L S_{A}} \underline{\hat{L}}_{(a} \hat{S}^{A}{ }_{b)},
\end{aligned}
$$

where we have defined the trace on the sphere,

$$
T^{\varnothing} \equiv \delta^{A B} T_{S_{A} S_{B}}
$$

and the tracefree part of the projection,

$$
T_{A B}^{\mathrm{TF}}=\perp_{A B}^{a b} T_{a b} .
$$

For this we employ the tracefree projection operator,

$$
\begin{aligned}
& \perp_{A B}^{a b}=\hat{S}_{A}{ }^{a} \hat{S}_{B}{ }^{b}-\frac{1}{2} \delta_{A B}\left(\delta^{C D} \hat{S}_{C}{ }^{a} \hat{S}_{D}{ }^{b}\right), \\
& \perp_{a b}^{A B}=\hat{S}_{a}^{A}{ }_{a} \hat{S}^{B}{ }_{b}-\frac{1}{2} \delta^{A B}\left(\delta_{C D} \hat{S}^{C}{ }_{a} \hat{S}^{D}{ }_{b}\right) .
\end{aligned}
$$

The independent components of $T_{A B}^{\mathrm{TF}}$ are

$$
\begin{aligned}
& T_{11}^{\mathrm{TF}}=-T_{22}^{\mathrm{TF}}=\frac{1}{2}\left(T_{S_{1} S_{1}}-T_{S_{2} S_{2}}\right) \equiv T^{+}, \\
& T_{12}^{\mathrm{TF}}=T_{S_{1} S_{2}} \equiv T^{\times} .
\end{aligned}
$$

They correspond of course to the two gravitational wave polarizations in the case of physical interest.

\section{THE ASYMPTOTIC SYSTEM FOR FIRST AND SECOND ORDER SYSTEMS}

\section{A. Second order systems}

Consider a set of quasilinear wave equations of the form

$$
\stackrel{\circ}{\square} u_{I}=\sum_{\substack{|\alpha| \leq|\beta| \leq 2,|\beta| \geq 1}} a_{I, \alpha \beta}^{J K} \partial^{\alpha} u_{J} \partial^{\beta} u_{K}+G\left(u, u^{\prime}, u^{\prime \prime}\right),
$$

where $I, J, K \in\{1, \ldots, n\}, u=\left(u_{1}, \ldots, u_{n}\right), \stackrel{\circ}{\square}=-\partial_{t}^{2}+$ $\delta^{i j} \partial_{i} \partial_{j}, x^{i}$ with $i, j \in\{1,2,3\}, \alpha, \beta$ are multi-indices and $G\left(u, u^{\prime}, u^{\prime \prime}\right)$ vanishes to third order as $\left(u, u^{\prime}, u^{\prime \prime}\right) \rightarrow 0$, with small initial data

$$
u(0, x)=v(x) \in C^{\infty}, \quad \partial_{T} u(0, x)=w(x) \in C^{\infty},
$$

decaying fast as $R \rightarrow \infty$, where $R=\sqrt{\delta_{i j} x^{i} x^{j}}$. Recall that a quadratic form $N_{a b} \equiv N\left(\stackrel{\circ}{\nabla}_{a} w, \stackrel{\circ}{\nabla}_{a} w\right)$ where $w$ is a tensor, is said to be a null form if it vanishes upon formal replacement of $\nabla_{a}^{\circ} w$ with $v_{a} w$, where $v_{a}$ is null. Similar definitions are made for quadratic forms involving higher derivatives. The set 13 is said to satisfy the null-condition if the quadratic nonlinearity is a null-form. The value of this definition is that, in $3+1$-dimensions, systems that satisfy the null-condition have global solutions when fed small data, and moreover have solutions of the same asymptotic behavior as a linear wave equation near null-infinity 31. The key subtlety is that the nullcondition is sufficient for long-term existence, but not necessary. For numerical applications the more relevant question concerns the asymptotic behavior of solutions rather than small-data global existence, but because of their close relationship in the mathematics literature they are often discussed in tandem. For example the weak null condition, originally introduced in 24, is crucially tied to the notion of the asymptotic system. One says that the wave equation (13) satisfies the weak null condition if the corresponding asymptotic system,

$$
\partial_{s} \partial_{q} U_{I}=A_{I, n m}^{J K}(\varpi) \partial_{q}^{m} U_{J} \partial_{q}^{n} U_{K}
$$

where

$$
\begin{gathered}
A_{I, n m}^{J K}(\varpi)=\sum_{|\alpha|=n,|\beta|=m} a_{I, \alpha \beta} \hat{\varpi}^{\alpha} \hat{\varpi}^{\beta}, \\
\hat{\varpi}=(-1, \varpi), \quad \varpi \in \mathbb{S}^{2},
\end{gathered}
$$


and $U_{i}=R u_{i}, q=R-T, s=\ln R$, has solutions which, roughly speaking, exist for all $s$, and whose derivatives grow at most exponentially in $s$ for all initial data decaying sufficiently fast in $q$. In this language, the classical null condition states that $A_{I, n m}^{J K}(\varpi)=0$. Thus, a system of quasilinear wave equations of the form given above satisfying the classical null condition will trivially satisfy the weak null condition, see [24, 32, 33] for further details. It is conjectured that systems satisfying the weak null condition admit small-data global solutions with asymptotics near null-infinity as predicted by the asymptotic system.

Model equation in second order form: An illustrative example is to consider the following model equation

$$
\stackrel{\circ}{\square} \phi=\left(\partial_{T} \tilde{\phi}\right)^{2}, \quad \stackrel{\circ}{\square} \tilde{\phi}=0 .
$$

where the flat wave operator $\square$ is expressed in spherical polar coordinates $\left(T, R, \theta^{A}\right)$. To derive the asymptotic system, define $\Phi=R \phi$ and $\tilde{\Phi}=R \tilde{\phi}$ and make the change of coordinates $\left(T, R, \theta^{A}\right) \rightarrow\left(q, s, \theta^{A}\right)$ where $q=R-T$ and $s=\ln R$. Substituting the expression for $\phi$ and $\tilde{\phi}$ in terms of the rescaled variables $\Phi$ and $\tilde{\Phi}$ into equation (17) and formally equating the terms with coefficients $R^{-2}$, one obtains the following asymptotic system

$$
\partial_{s} \partial_{q} \Phi=\left(\partial_{q} \tilde{\Phi}\right)^{2}, \quad \partial_{s} \partial_{q} \tilde{\Phi}=0
$$

Equation (17) is an example of a system of equations that does not satisfy the classical null condition but satisfies the weak null condition. Observe that the second equation in $(18)$ simply states that $\partial_{q} \tilde{\Phi}$ does not depend on $s$. Then, using the method of characteristics, one can integrate in $s$ the first equation in 18 to conclude that $\partial_{q} \Phi=s\left(\partial_{q} \tilde{\Phi}\right)^{2}$. The latter implies for the original set of variables that $\partial \phi$ decays as $R^{-1} \ln R 24$.

The motivation for the introduction of the asymptotic system is related to the observation that one expects better decay rates for $L$-derivatives of the field compared with other derivatives. Therefore, the construction of the asymptotic system is essentially to rescale the raw variables according to their expected fall-off, move to suitable coordinates, and then to throw away all quadratic terms containing derivatives containing at least one derivative tangential to the outgoing light-cone, along with cubic and higher order terms. We will see in the next section how each of these ingredients is adjusted for first order systems.

\section{B. First order systems}

To the best of our knowledge, the notion of the weak null condition and the asymptotic systems has been exploited only for systems of wave equations. In the context of gravitation, in particular, for the Einstein field equations in harmonic gauge in 34] and more general systems of wave equations in [25]. Although the wave equation can be recast straightforwardly as a first order symmetric hyperbolic system by the introduction of an order reduction variable $w=\partial u$, the weak null condition and the notion of the asymptotic system has not been discussed in this context yet. This is of importance for applications since numerical evolution schemes often make use of a first order formulation of the field equations. Moreover, although mathematically, the relation $w=\partial u$ connects the first and second order formulations, numerically, the use of reduction variable requires the introduction of a reduction constraint $\mathcal{C} \equiv w-\partial u$, and violations to this reduction constraint tends to grow exponentially, or worse, during numerical evolutions [26 28, 35. Therefore, one is forced to modify the evolution equations to control this bad behavior. With this motivation in mind, in this section, the model equation 17 is written in a form that is suitable for numerical implementations and then the corresponding asymptotic system is derived.

Model equation in first order form: Let $\left(\stackrel{\mathcal{M}}{,} m_{a b}\right)$ denote the Minkowski spacetime equipped with the objects introduced in section $\mathrm{II}$ and consider the model equation (17). Let $N^{a}$ denote the time unit normal to the surface determined by the condition $T=0$ and define $\stackrel{\circ}{\gamma}_{a b}=m_{a b}+\stackrel{\circ}{N}_{a} \stackrel{\circ}{N}_{b}$. Let $\phi_{a}$ be a spatial covector, namely $N^{a} \phi_{a}=0$, so that $\phi_{R}$ and $\phi_{S_{A}}$ denote the components of $\phi_{a}$ respect to the spatial frame $\left(\left(\partial_{R}\right)^{a}, S_{A}{ }^{a}\right)$. To perform the order reduction, define the reduction constraints as

$$
\mathcal{C}_{a} \equiv \stackrel{\circ}{\gamma}_{a}^{b} \stackrel{\circ}{\nabla}_{b} \phi-\phi_{a},
$$

and the time reduction variable $\pi$ via $\pi=-\partial_{T} \phi$. With formally identical definitions for $\tilde{\phi}$, the evolution equations for these fields read,

$$
\begin{aligned}
& \partial_{T} \tilde{\phi}=-\tilde{\pi}, \\
& \partial_{T} \tilde{\phi}_{R}=-\partial_{R} \tilde{\pi}+\gamma_{2}\left(-\tilde{\phi}_{R}+\partial_{R} \tilde{\phi}\right), \\
& \partial_{T} \tilde{\phi}_{S_{A}}=\gamma_{2}\left(-\tilde{\phi}_{S_{A}}+S_{A}^{a} \partial_{a} \tilde{\phi}\right)-S_{A}^{b} \partial_{b} \tilde{\pi}, \\
& \partial_{T} \tilde{\pi}=-\delta^{A B} S_{A}{ }^{a} \partial_{a} \tilde{\phi}_{S_{B}}-\partial_{R} \tilde{\phi}_{R}-\frac{2}{R} \tilde{\phi}_{R}-\tilde{\phi}_{S_{A}} \stackrel{\circ}{\nabla}^{a} S_{a}^{A}, \\
& \partial_{T} \phi=-\pi, \\
& \partial_{T} \phi_{R}=-\partial_{R} \pi+\gamma_{2}\left(-\phi_{R}+\partial_{R} \phi\right), \\
& \partial_{T} \phi_{S_{A}}=\gamma_{2}\left(-\phi_{S_{A}}+S_{A}{ }^{a} \partial_{a} \phi\right)-S_{A}{ }^{b} \partial_{b} \pi \text {, } \\
& \partial_{T} \pi=\tilde{\pi}^{2}-\delta^{A B} S_{A}{ }^{a} \partial_{a} \phi_{S_{B}}-\partial_{R} \phi_{R}-\frac{2}{R} \phi_{R} \\
& -\phi_{S_{A}} \stackrel{\circ}{\nabla}^{a} S_{a}^{A} \text {. }
\end{aligned}
$$

The evolution equations for $\phi$ and $\tilde{\phi}$ in 20 are just the definition of the time reduction variables. The evolution equations for $\phi_{R}, \phi_{S_{A}}$ and the corresponding hatted variables in 20 with $\gamma_{2}=0$ arise as a consequence of the no-torsion condition $\left[\stackrel{\circ}{\nabla}_{a}, \stackrel{\circ}{\nabla}_{b}\right] \phi=0$ and $\left[\stackrel{\circ}{\nabla}_{a}, \stackrel{\circ}{\nabla}_{b}\right] \tilde{\phi}=0$. Nevertheless, to reduce the effect produced by constraint violations in numerical applications, one modifies these equations by adding the following multiples of the reduction constraints: $\gamma_{2} \mathcal{C}_{S_{A}}, \gamma_{2} \mathcal{C}_{R}, \gamma_{2} \tilde{\mathcal{C}}_{S_{A}}$, and $\gamma_{2} \tilde{\mathcal{C}}_{R}$. Here $\gamma_{2}$ is a freely prescribable scalar function of the coordinates. Notice that the introduction of these terms affect the principal part of the equation. Nonetheless, one can show 
that this system is symmetric hyperbolic for any choice of the formulation parameter $\gamma_{2}$ [28]. The evolution equations for $\pi$ and $\tilde{\pi}$ in 20 arise from expressing the wave equations (17) using the reduction variables.

Following the discussion of the asymptotic system of section III A, one needs to rescale the variables appropriately. Nevertheless, since in the first order reduction of the equations the frame $\left(\left(\partial_{T}\right)^{a},\left(\partial_{R}\right), S_{A}{ }^{a}\right)$ was used to express the components of the reduction variable instead of the flat null frame, one needs to perform the following change of variables before rescaling. Defining

$$
\sigma^{+}=-\pi+\phi_{R}, \quad \sigma^{-}=-\pi-\phi_{R},
$$

with formally identical definitions for the hatted variables, substituting $\pi, \phi_{R}, \tilde{\pi}$ and $\tilde{\phi}_{R}$ written in terms of $\sigma^{+}, \sigma^{-}, \tilde{\sigma}^{+}$and $\tilde{\sigma}^{-}$into equation 20 one obtains a set of evolution equations for the variables,

$$
\left\{\phi, \quad \sigma^{+}, \quad \sigma^{-}, \quad \phi_{S_{A}}, \quad \tilde{\phi}, \quad \tilde{\sigma}^{+}, \quad \tilde{\sigma}^{-}, \quad \tilde{\phi}_{S_{A}}\right\} .
$$

Observe that when the constraints are satisfied $\sigma^{+}$ and $\sigma^{-}$correspond to the $L$ and $\underline{L}$ derivatives of $\phi$, respectively. Then, one defines the rescaled variables as,

$$
\begin{aligned}
\Phi & =R \phi, & \Sigma^{+} & =R^{2} \sigma^{+}, \\
\Sigma^{-} & =R \sigma^{-}, & \Phi_{S_{A}} & =R^{2} \phi_{S_{A}},
\end{aligned}
$$

along with the analogous expressions for the hatted variables, and substitutes these definitions into the evolution equations using the chain rule to express the derivatives in terms of $\partial_{q}$ and $\partial_{s}$. Notice the important point that the rescaling here takes place after the derivative is applied. It is easily seen that this is the natural construction by considering spherically symmetric solutions to the flat-space wave equation. Solving for the derivatives of the rescaled variables we obtain,

$$
\begin{aligned}
\partial_{q} \tilde{\Sigma}^{+} & \simeq \frac{1}{2} \tilde{\Sigma}^{-}+\frac{1}{2} \gamma_{2}\left(\tilde{\Sigma}^{+}+\tilde{\Phi}-\partial_{s} \tilde{\Phi}\right), \\
\partial_{s} \tilde{\Sigma}^{-} & \simeq \gamma_{2}\left(\tilde{\Sigma}^{+}+\tilde{\Phi}-\partial_{s} \tilde{\Phi}\right), \\
\partial_{q} \tilde{\Phi}_{S_{A}} & \simeq-\frac{1}{2} \omega_{A}{ }^{a} \partial_{a} \tilde{\Sigma}^{-}+\gamma_{2}\left(\tilde{\Phi}_{S_{A}}-\omega_{A}{ }^{a} \partial_{a} \tilde{\Phi}\right), \\
\partial_{q} \tilde{\Phi} & \simeq-\frac{1}{2} \tilde{\Sigma}^{-}, \\
\partial_{q} \Sigma^{+} & \simeq \frac{1}{8}\left(4 \Sigma^{-}+\left(\tilde{\Sigma}^{-}\right)^{2}\right)+\frac{1}{8} \gamma_{2}\left(4 \Sigma^{+}+4 \Phi-4 \partial_{s} \Phi\right), \\
\partial_{s} \Sigma^{-} & \simeq-\frac{1}{4}\left(\tilde{\Sigma}^{-}\right)^{2}+\gamma_{2}\left(\Sigma^{+}+\Phi-\partial_{s} \Phi\right), \\
\partial_{q} \Phi_{S_{A}} & \simeq-\frac{1}{2} \omega_{A}{ }^{a} \partial_{a} \Sigma^{-}+\gamma_{2}\left(\Phi_{S_{A}}-\omega_{A}{ }^{a} \partial_{a} \Phi\right), \\
\partial_{q} \Phi & \simeq-\frac{1}{2} \Sigma^{-},
\end{aligned}
$$

where $\simeq$ represents equality up to error terms which decay one order faster in $R$ than the displayed expressions. In this case, it represents equality up to order $O\left(R^{-1}\right)$. These expressions are derived in full in the mathematica notebooks associated with this paper 36. They require xAct 37.

Neglecting the error terms implicit in the last equations, namely, formally replacing $\simeq$ with $=$ defines the asymptotic system for equation (20). To see that this actually corresponds to the asymptotic system 18 one has to examine the relation between the rescaled variables and the corresponding rescaled reduction constraints. Let,

$$
\mathbb{C}_{R}=R^{2} \mathcal{C}_{R}, \quad \mathbb{C}_{S_{A}}=R^{2} \mathcal{C}_{S_{A}},
$$

with analogous definitions for the hatted reduction constraints. A direct computation using the first and fifth equation in (20), equation (19), and the definitions for the rescaled variables give,

$$
\begin{aligned}
\Sigma^{+} & =-\mathbb{C}_{R}-\Phi+\partial_{s} \Phi, \\
\Sigma^{-} & =-2 \partial_{q} \Phi+\frac{\mathbb{C}_{R}}{R}+\frac{\Phi}{R}-\frac{\partial_{s} \Phi}{R}, \\
\Phi_{S_{A}} & =-\mathbb{C}_{S_{A}}+\omega_{A}{ }^{a} \partial_{a} \Phi,
\end{aligned}
$$

and identical expressions for the hatted rescaled variables. Substituting (25) and the corresponding hatted version of these expressions into equation (23) and rearranging one obtains,

$$
\begin{aligned}
2 \partial_{s} \partial_{q} \Phi & \simeq \frac{1}{4}\left(\tilde{\Sigma}^{-}\right)^{2}+\gamma_{2} \mathbb{C}_{R}, & 2 \partial_{s} \partial_{q} \tilde{\Phi} & \simeq \tilde{\gamma}_{2} \tilde{\mathbb{C}}_{R}, \\
\partial_{q} \Phi & \simeq-\frac{1}{2} \Sigma^{-}, & \partial_{q} \tilde{\Phi} & \simeq-\frac{1}{2} \tilde{\Sigma}^{-}, \\
\partial_{q} \mathbb{C}_{R} & \simeq \gamma_{2} \mathbb{C}_{R}, & \partial_{q} \tilde{\mathbb{C}}_{R} & \simeq \gamma_{2} \tilde{\mathbb{C}}_{R}, \\
\partial_{q} \mathbb{C}_{S_{A}} & \simeq \gamma_{2} \mathbb{C}_{S_{A}}, & \partial_{q} \tilde{\mathbb{C}}_{S_{A}} & \simeq \gamma_{2} \tilde{\mathbb{C}}_{S_{A}} .
\end{aligned}
$$

These expressions are valid for any choice of the damping parameter $\gamma_{2}$. Observe that if this parameter is set to zero or, more generally are chosen to decay sufficiently fast, say $\gamma_{2} \simeq R^{-1}$, then one has

$$
\begin{aligned}
2 \partial_{s} \partial_{q} \Phi & \simeq \frac{1}{4}\left(\tilde{\Sigma}^{-}\right)^{2}, & 2 \partial_{s} \partial_{q} \tilde{\Phi} & \simeq 0, \\
\partial_{q} \Phi+\frac{1}{2} \Sigma^{-} & \simeq 0, & \partial_{q} \tilde{\Phi}+\frac{1}{2} \tilde{\Sigma}^{-} & \simeq 0, \\
\partial_{q} \mathbb{C}_{R} & \simeq 0, & \partial_{q} \tilde{\mathbb{C}}_{R} & \simeq 0, \\
\partial_{q} \mathbb{C}_{S_{A}} & \simeq 0, & \partial_{q} \tilde{\mathbb{C}}_{S_{A}} & \simeq 0 .
\end{aligned}
$$

Following the philosophy of the asymptotic system by formally replacing $\simeq$ with $=$ one realizes that the last four expressions in (27) can be regarded as the asymptotic equations for the rescaled reduction constraints. Integrating these equations, along an integral curve of $\partial_{q}$, from a fixed $q_{\star}$ to $q$ reveals,

$$
\mathbb{C}_{R}=\mathbb{C}_{R}^{\star}, \quad \mathbb{C}_{S_{A}}=\mathbb{C}_{S_{A}}^{\star} .
$$

where $\mathbb{C}_{R}^{\star}=\left.\mathbb{C}_{R}\right|_{q=q_{\star}}, \mathbb{C}_{S_{A}}^{\star}=\left.\mathbb{C}_{S_{A}}\right|_{q=q_{\star}}$ and similarly for the hatted rescaled reduction constraints. On the other hand, the first four equations in 27) simply encode

$$
\partial_{s} \partial_{q} \Phi=\left(\partial_{q} \tilde{\Phi}\right)^{2}, \quad \partial_{s} \partial_{q} \tilde{\Phi}=0
$$

Integrating these equations, this time along an integral curve of $\partial_{s}$, we find,

$$
\Phi=s \mathcal{G}_{\Phi}\left(q, \theta^{A}\right), \quad \tilde{\Phi}=\mathcal{G}_{\tilde{\Phi}}\left(q, \theta^{A}\right),
$$

where $\mathcal{G}_{\Phi}$ and $\mathcal{G}_{\tilde{\Phi}}$ are regular functions of their arguments. Since we are only interested in the behavior of 
the fields for large $R$, to have a more compact notation, the symbol $\sim$ will be used to denote equality where the functional dependence on the other coordinates has been suppressed. Consistent with this notation one writes,

$$
\Phi \sim \ln R, \quad \tilde{\Phi} \sim 1 .
$$

Assuming $\mathbb{C}_{R}^{\star}, \mathbb{C}_{S_{A}}^{\star}, \tilde{\mathbb{C}}_{R}^{\star}$ and $\tilde{\mathbb{C}}_{S_{A}}^{\star}$ are uniformly bounded, then, using equations 25 one has that,

$$
\begin{aligned}
& \Sigma^{+} \sim \ln R, \quad \Sigma^{-} \sim \ln R, \quad \Phi_{S_{A}} \sim \ln R, \\
& \tilde{\Sigma}^{+} \sim 1, \quad \tilde{\Sigma}^{-} \sim 1, \quad \tilde{\Phi}_{S_{A}} \sim 1 .
\end{aligned}
$$

Therefore one concludes that,

$$
\begin{aligned}
\sigma^{+} \sim \frac{\ln R}{R^{2}}, & \sigma^{-} \sim \frac{\ln R}{R}, & \phi_{S_{A}} \sim \frac{\ln R}{R^{2}}, \\
\tilde{\sigma}^{+} \sim \frac{1}{R^{2}}, & \tilde{\sigma}^{-} \sim \frac{1}{R}, & \tilde{\phi}_{S_{A}} \sim \frac{1}{R^{2}} .
\end{aligned}
$$

Clearly if we wished to perform numerical evolution of this system on a compactified domain including nullinfinity this result tells us that the rescaling of variables resulting in $\Sigma^{-}$would be too aggressive, since we need that the evolved variables are at least not divergent.

\section{A CONSTRAINT DAMPED HYPERBOLIC REDUCTION OF GR IN SECOND ORDER FORM}

\section{A. Hyperbolic reductions of GR}

Let $\left(\mathcal{M}, g_{a b}\right)$ denote a 4-dimensional manifold equipped with a metric $g_{a b}$. Let $m_{a b}$ denote the Minkowski metric and let $\nabla$ and $\nabla^{\circ}$ denote the LeviCivita connection of $g_{a b}$ and $m_{a b}$, respectively. The relation between $\nabla$ and $\nabla$ can be parameterized via,

$$
\nabla_{a} v_{b}=\stackrel{\circ}{\nabla}_{a} v_{b}-\Gamma_{a b}^{c} v_{c}
$$

where $v_{a}$ is any covector. This relation can be taken to define $\Gamma_{a b}^{c}$. Consequently $\Gamma_{a b}^{c}$ can be expressed in terms

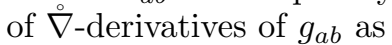

$$
\Gamma_{a b}^{c}=\frac{1}{2} g^{c d}\left(\stackrel{\circ}{\nabla}_{a} g_{b d}+\stackrel{\circ}{\nabla}_{b} g_{a d}-\stackrel{\circ}{\nabla}_{d} g_{a b}\right) .
$$

Defining the contracted Christoffel symbols via $\Gamma^{c} \equiv$ $g^{a b} \Gamma_{a b}^{c}$, the Ricci tensor can be compactly expressed as,

$$
\begin{aligned}
R_{a b}= & -\frac{1}{2} g^{c d} \stackrel{\circ}{\nabla}_{c} \stackrel{\circ}{\nabla}_{d} g_{a b}+\nabla_{(a} \Gamma_{b)} \\
& +g^{c d} g^{h f}\left(\Gamma_{b d f} \Gamma_{c a h}+2 \Gamma_{d b f} \Gamma_{(a c) h}\right) .
\end{aligned}
$$

Let $C^{a} \equiv F^{a}+\Gamma^{a}$ where $F^{a}$ are smooth functions of the coordinates $X^{\mu}$ and the metric $g_{a b}$ but not its derivatives. These are known as the gauge source functions as a choice of $F^{a}$ determines a coordinate system $X^{\mu}$. To see this, observe that $\Gamma^{\mu}=-\nabla^{\nu} \nabla_{\nu} X^{\mu}$, thus requiring $C^{\mu}=0$ is equivalent to

$$
\nabla^{\nu} \nabla_{\nu} X^{\mu}=F^{\mu}
$$

The equations $C^{a}=0$ will be called GHG or harmonic constraints in the $F^{a}=0$ case. If they are satisfied then, using equation (36), one sees that the vacuum Einstein field equations reduce to a set of wave equations for $g_{a b}$

$$
\begin{aligned}
g^{c d} \stackrel{\circ}{\nabla}_{c} \stackrel{\circ}{\nabla}_{d} g_{a b}= & 2 g^{c d} g^{h f}\left(\Gamma_{b d f} \Gamma_{c a h}+2 \Gamma_{d b f} \Gamma_{(a c) h}\right) \\
& +2 \nabla_{(a} F_{b)},
\end{aligned}
$$

where $\Gamma_{a b c}$ is expressed in terms of derivatives of the metric $g_{a b}$ using equation (35). This hyperbolic reduction process can be succinctly expressed as follows. Define the reduced Ricci tensor as

$$
\underline{R}_{a b}=R_{a b}-\nabla_{(a} C_{b)},
$$

so that equation $(38)$ is encoded in the condition $\underline{R}_{a b}=$ 0 . Observe that if the constraint $C^{a}=0$ is satisfied then $\underline{R}_{a b}=R_{a b}$. Moreover, if $\underline{R}_{a b}=0$ then, as a consequence of the contracted second Bianchi identity $\nabla^{a} R_{a b}-\frac{1}{2} \nabla_{b} R=0$, one has that $C_{a}$ satisfies the following propagation equation

$$
\nabla^{a} \nabla_{a} C_{b}=-C^{a} \nabla_{(a} C_{b)} .
$$

Since this is a wave equation homogeneous in $C_{a}$, the latter implies that if $C_{a}$ and $\nabla_{a} C_{b}$ vanish on a spacelike hypersurface $\mathcal{S} \subset \mathcal{M}$ then $C_{a}=0$ in $\mathcal{D}(\mathcal{S}) \subset \mathcal{M}$ [38, 39]. Here the domain of dependence of an achronal set $\mathcal{A}$, is denoted as $\mathcal{D}(\mathcal{A})$. Observe that this hyperbolic reduction strategy is not unique as one can define a reduced Ricci tensor as

$$
\underline{R}_{a b}=R_{a b}-\nabla_{(a} C_{b)}+\mathcal{T}_{a b} .
$$

where $\mathcal{T}_{a b}$ is any expression homogeneous in $C_{a}$ so that $C_{a}=0$ implies $\underline{R}_{a b}=R_{a b}$. The corresponding propagation equation for $C_{a}$ is then,

$$
\nabla^{a} \nabla_{a} C_{b}=-C^{a} \nabla_{(a} C_{b)}+2 \nabla^{a} \mathcal{T}_{a b}^{\mathrm{TR}}+C^{a} \mathcal{T}_{b a},
$$

where $\mathcal{T}_{a b}^{\mathrm{TR}} \equiv \mathcal{T}_{a b}-\frac{1}{2} g_{a b} \mathcal{T}_{c}^{c}$. Observe that, the right hand side of the last equation is homogeneous in $C_{a}$ as long as $\mathcal{T}_{a b}$ is chosen to be homogeneous in $C_{a}$. Although all the possible reduced equations $\underline{R}_{a b}=0$ are equivalent if the GHG constraints are satisfied, a different choice of $\mathcal{T}_{a b}$ can be used to obtain equations of a particular desired form. For instance in 27, the constraint addition is chosen as $\mathcal{T}_{a b}=\gamma \mathcal{T}_{a b}$ where,

$$
{ }^{\gamma} \mathcal{T}_{a b}=\gamma_{4} \Gamma^{c}{ }_{a b} C_{c}-\frac{1}{2} \gamma_{5} g_{a b} \Gamma^{e} C_{e}-\gamma_{0}\left(n_{(a} C_{b)}-g_{a b} n^{c} C_{c}\right),
$$

here $n_{a}$ is a freely specifiable vector and $\gamma_{0}, \gamma_{4}, \gamma_{5}$ are, in general, scalar functions depending on the coordinates. The parameter $\gamma_{0}$ is included to damp away high frequency constraint violations while the parameters $\gamma_{4}$ and $\gamma_{5}$ are included to modify $\nabla_{(a} C_{b}$ so the constraint addition made in the construction of the formulation is done either respect to $\nabla$ or $\nabla$, or some combination thereof. 
Other choices are designed instead to exhibit certain structures of the equations. In particular, if one sets $\mathcal{T}_{a b}={ }^{\mathcal{C}} \mathcal{T}_{a b}$ where

$$
{ }^{\mathcal{C}} \mathcal{T}_{a b}=\frac{1}{2} C_{a} C_{b}-C^{c} \stackrel{\circ}{\nabla}_{(a} g_{b) c} .
$$

Then the reduced Einstein field equations $\underline{R}_{a b}=0$ read

$$
g^{c d} \stackrel{\circ}{\nabla}_{d} \stackrel{\circ}{\nabla}_{c} g_{a b}=P_{a b}[\stackrel{\circ}{\nabla} g]+Q_{a b}[\stackrel{\circ}{\nabla} g]+F_{a b},
$$

with

$$
\begin{aligned}
P_{a b}[\stackrel{\nabla}{\nabla} g] & =-\frac{1}{2} g^{c d} g^{f h} \stackrel{\circ}{\nabla}_{a} g_{c f} \stackrel{\circ}{\nabla}_{b} g_{d h}+\frac{1}{4} g^{c d} g^{f h} \stackrel{\circ}{\nabla}_{a} g_{c d} \stackrel{\circ}{\nabla}_{b} g_{f h}, \\
Q_{a b}[\stackrel{\circ}{\nabla} g] & =-g^{c d} g^{f h}\left(\frac{1}{2} \stackrel{\circ}{\nabla}_{b} g_{f h} \stackrel{\circ}{\nabla}_{d} g_{a c}-\frac{1}{2} \stackrel{\circ}{\nabla}_{a} g_{f h} \stackrel{\circ}{\nabla}_{d} g_{b c}\right. \\
& +\frac{1}{2} \stackrel{\circ}{\nabla}_{a} g_{b c} \stackrel{\circ}{\nabla}_{d} g_{f h}+\frac{1}{2} \stackrel{\circ}{\nabla}_{b} g_{a c} \stackrel{\circ}{\nabla}_{d} g_{f h}+\stackrel{\circ}{\nabla}_{b} g_{d h} \stackrel{\circ}{\nabla}_{f} g_{a c} \\
& -\stackrel{\circ}{\nabla}_{d} g_{b h} \stackrel{\circ}{\nabla}_{f} g_{a c}+\stackrel{\circ}{\nabla}_{a} g_{d h} \stackrel{\circ}{\nabla}_{f} g_{b c}+\stackrel{\circ}{\nabla}_{f} g_{a c} \stackrel{\circ}{\nabla}_{h} g_{b d} \\
& \left.+\stackrel{\circ}{\nabla}_{d} g_{a c} \stackrel{\circ}{\nabla}_{h} g_{b f}-\stackrel{\circ}{\nabla}_{a} g_{b c} \stackrel{\circ}{\nabla}_{h} g_{d f}-\stackrel{\circ}{\nabla}_{b} g_{a c} \stackrel{\circ}{\nabla}_{h} g_{d f}\right),
\end{aligned}
$$

and the gauge source functions $F^{a}$ appear in the form,

$$
F_{a b}=2 \nabla_{(a} F_{b)}-F_{a} F_{b}+2 F^{c} \stackrel{\circ}{\nabla}_{(a} g_{b) c} .
$$

The relevant observation here is that $Q_{a b}$ is a null form. Therefore, taking $F^{a}=0$, the constraint addition (44) places the reduced field equations $\underline{R}_{a b}=0$ in the form given in 32 where the global stability of the Minkowski spacetime in wave coordinates was proven for a set of restricted initial data, when the harmonic constraints are fulfilled.

\section{B. The asymptotic system and the weak null condition}

In this section the asymptotic system for the Einstein field equations in harmonic gauge as discussed in [24, 32, 33 is reviewed in our conventions and the problems that the above hyperbolic reductions present for free evolution schemes in NR are discussed.

Consider a perturbation of the Minkowski spacetime, and write $g_{a b}$ as

$$
g_{a b}=m_{a b}+h_{a b},
$$

where $h_{a b}$ is a symmetric 2-tensor. For the discussion in the remainder of this subsection all the indices are moved using the flat metric $m_{a b}$ except for $g_{a b}$ for which we have

$$
g^{a b}=m^{a b}-h^{a b}+O^{a b}\left(h^{2}\right),
$$

where $h^{a b}=m^{a c} m^{d b} h_{c d}$ and $O^{a b}\left(h^{2}\right)$ vanishes to second order at $h=0$ 34. This convention for raising and lowering indices will be used when discussing the derivation of the asymptotic system of a given set of equations but it will be avoided otherwise. Now, consider the reduced Ricci operator $\underline{R}_{a b}=R_{a b}-\nabla_{(a} C_{b)}+\mathcal{T}_{a b}$ with $\mathcal{T}_{a b}={ }^{\mathcal{C}} \mathcal{T}_{a b}$ as in equation (44) in harmonic gauge, in other words, with vanishing gauge source functions $F^{a}=0$. In this case, the reduced Einstein field equations $\underline{R}_{a b}=0$ imply the following system of wave equations for $g_{a b}$

$$
g^{c d} \stackrel{\circ}{\nabla}_{d} \stackrel{\circ}{\nabla}_{c} g_{a b}=P_{a b}[\stackrel{\circ}{\nabla} g]+Q_{a b}[\stackrel{\circ}{\nabla} g] .
$$

Using equation 49 and (50) one obtains the following evolution equations for the perturbation $h_{a b}$

$$
g^{c d} \stackrel{\circ}{\nabla}_{c} \stackrel{\circ}{\nabla}_{d} h_{a b}=P_{a b}[\stackrel{\circ}{\nabla} h]+Q_{a b}[\stackrel{\circ}{\nabla} h]+O_{a b}\left(h\left(\stackrel{\circ}{\nabla}^{2} h\right)^{2}\right),
$$

with $P_{a b}\left[{ }^{\nabla} h\right]$ and $Q_{a b}[\stackrel{\circ}{\nabla} h]$ as given in equations $(46)-(47)$ where $\nabla^{\circ} g$ is formally replaced by $\stackrel{\circ}{\nabla}^{\circ} h$ and $O_{a b}\left(h(\nabla h)^{2}\right)$ denotes a quadratic form in $\stackrel{\circ}{\nabla}_{a} h_{b c}$ with coefficients depending on $h_{a b}$ which vanish for $h_{a b}=0$. Similarly, the harmonic coordinate condition reads

$$
m^{a c} \stackrel{\circ}{\nabla}_{c} h_{a b}=\frac{1}{2} m^{a c} \stackrel{\circ}{\nabla}_{b} h_{a c}+O_{b}(h \stackrel{\circ}{\nabla} h),
$$

where $O_{b}(h \stackrel{\circ}{\nabla} h)$ is linear in $\stackrel{\circ}{\nabla}_{a} h_{b c}$ with coefficients depending on $h_{a b}$ which vanish for $h_{a b}=0$. To derive the asymptotic system, let

$$
q=R-T, \quad s=\ln R, \quad H_{a b}=R h_{a b} .
$$

To have a more compact notation for directional derivatives that will often appear, the following notation will be used,

$$
\begin{array}{ll}
\stackrel{\circ}{\nabla}_{T}=\left(\partial_{T}\right)^{a} \stackrel{\circ}{\nabla}_{a}, & \stackrel{\circ}{\nabla}_{R}=\left(\partial_{R}\right)^{a} \stackrel{\circ}{\nabla}_{a}, \\
\stackrel{\circ}{\nabla}_{q}=\left(\partial_{q}\right)^{a} \stackrel{\circ}{\nabla}_{a}, & \stackrel{\circ}{\nabla}_{s}=\left(\partial_{s}\right)^{a} \stackrel{\circ}{\nabla}_{a} .
\end{array}
$$

Substituting (49) into 45), re-expressing the equation in terms of $H_{a b}$ and formally equating terms with coefficients $R^{-2}$, one obtains the following asymptotic system

$$
\left(2 \stackrel{\circ}{\nabla}_{s}-H_{L L} \stackrel{\circ}{\nabla}_{q}\right) \stackrel{\circ}{\nabla}_{q} H_{a b}=\hat{L}_{a} \hat{L}_{b} P\left(\stackrel{\circ}{\nabla}_{q} H, \stackrel{\circ}{\nabla}_{q} H\right),
$$

where $H_{L L}=L^{a} L^{b} H_{a b}$ and

$$
\begin{aligned}
P\left(\stackrel{\circ}{\nabla}_{q} H\right)= & -\frac{1}{2} m^{a b} m^{c d} \stackrel{\circ}{\nabla}_{q} H_{a c} \stackrel{\circ}{\nabla}_{q} H_{b d} \\
& +\frac{1}{4} m^{a b} m^{c d} \stackrel{\circ}{\nabla}_{q} H_{a b} \stackrel{\circ}{\nabla}_{q} H_{c d} .
\end{aligned}
$$

Contracting equations with the flat null frame $\left(L^{a}, \underline{L}^{a}, S_{A}{ }^{a}\right)$, exploiting that

$$
\begin{aligned}
v^{a} w^{b} \stackrel{\circ}{\nabla}_{c} H_{a b} & \simeq \stackrel{\circ}{\nabla}_{c} H_{v w}, \\
v^{a} w^{b} \stackrel{\circ}{\nabla}_{s} \stackrel{\circ}{\nabla}_{q} H_{a b} & \simeq \partial_{s} \partial_{q} H_{v w}, \\
v^{a} w^{b} \stackrel{\circ}{\nabla}_{q} \stackrel{\circ}{\nabla}_{q} H_{a b} & \simeq \partial_{q} \partial_{q} H_{v w},
\end{aligned}
$$

for $v^{a}, w^{a} \in\left\{L^{a}, \underline{L}^{a}, S_{A}{ }^{a}\right\}$, one observes that the only equation in $(56)$ with a non-vanishing right-hand side is,

$$
\begin{aligned}
\left(2 \partial_{s}-H_{L L} \partial_{q}\right) \partial_{q} H_{\underline{L L}} & =-4\left(\partial_{q} H^{+}\right)^{2}-4\left(\partial_{q} H^{\times}\right)^{2} \\
& -\partial_{q} H_{\underline{L L}} \partial_{q} H_{L L}-2\left(\partial_{q} H_{L \underline{L}}\right)\left(\partial_{q} H^{\varnothing}\right) \\
& +4 \delta^{A B}\left(\partial_{q} H_{L S_{A}}\right)\left(\partial_{q} H_{\underline{L} S_{B}}\right),
\end{aligned}
$$


while all the others satisfy,

$$
\left(2 \partial_{s}-H_{L L} \partial_{q}\right) \partial_{q} H_{\mathcal{T} U}=0,
$$

where $\mathcal{T} \in\left\{L, S_{A}\right\}$ and $U \in\left\{L, \underline{L}, S_{A}\right\}$. Proceeding similarly for the harmonic coordinate condition

$$
m_{a b} C^{b}=m_{a b} \Gamma^{b}=m_{a b} F^{b}=0,
$$

using equations (49) and (54), and formally equating terms with coefficients $R^{-1}$, the asymptotic harmonic coordinate condition reads

$$
\stackrel{\circ}{\nabla}_{q} H_{L a}-\frac{1}{2} \hat{L}_{a} \stackrel{\circ}{\nabla}_{q} H=0,
$$

where $H_{L a}=L^{c} H_{c a}$ and $H=m^{a b} H_{a b}$.

Expressing equation 62 in components respect to the flat null frame one has,

$$
\partial_{q} H_{L \mathcal{T}}=0, \quad \partial_{q} H^{\varnothing}=0,
$$

where $H^{\varnothing}$ is defined according to equation (9). The asymptotic harmonic condition $(62)$ will play an important role in the subsequent discussion since in free evolution schemes in NR one cannot ensure that the constraint equations $C^{a}=0$ are satisfied but only that such violations are small. Moreover, notice that the asymptotic equation (60) implies that $\partial_{q} H_{\mathcal{T} U}$ is constant along the integral curves of the vector field $2 \partial_{s}-H_{L L} \partial_{q}$. Observing that constraint violations $C^{a} \neq 0$ imply that $\left.\left(\partial_{q} H_{L \mathcal{T}}\right)\right|_{\Sigma} \neq 0$ one concludes that, if constraint violations are present, they will be preserved in the asymptotic region along the integral curves of $2 \partial_{s}-H_{L L} \partial_{q}$. As an additional remark, observe that, if the constraints $C^{a}=0$ are satisfied, then one can exploit equations 63 to reduce the asymptotic equation $\sqrt{59}$ to

$$
\left(2 \partial_{s}-H_{L L} \partial_{q}\right) \partial_{q} H_{\underline{L L}}=-4\left(\partial_{q} H^{+}\right)^{2}-4\left(\partial_{q} H^{\times}\right)^{2},
$$

where $H^{\times}$and $H^{+}$correspond to the two gravitational wave polarizations as in equation 12 . This further simplification in the only equation of the asymptotic system (56) with non-vanishing right-hand side cannot be directly attained in free evolution form without modifying the hyperbolic reduction determined by the constraint addition ${ }^{\mathcal{C}} \mathcal{T}_{a b}$. Thus, one requires a hyperbolic reduction for which one recovers the latter equation without assuming that the constraint equations $(63)$ are fulfilled and, more importantly, a hyperbolic reduction for which constraint violations $C^{a} \neq 0$ are damped close to null infinity.

\section{The coordinate light-speed condition}

In this subsection asymptotic expressions for quantities relevant for the dual foliation formulation are written in terms of the variables of the asymptotic system. In particular, we are interested in finding how the solution to the asymptotic system determines the radial coordinate light-speed asymptotically near null-infinity. To connect the current discussion with the dual foliation formalism, is convenient to perform a $2+1+1$ split. Since in this subsection we are not computing the asymptotic system we will use the metric $g_{a b}$ - as usual - to raise and lower indices and not $m_{a b}$. In the language of [23] let $\left(T, R, \theta^{A}\right)$ be the upper case coordinate system $X^{\mu}$ and use $T$ to define the usual lapse, projection operator, normal and shift vectors

$$
\begin{aligned}
\mathcal{A} & \equiv\left(-g^{a b} \nabla_{a} T \nabla_{a} T\right)^{-1 / 2}, & N_{a} & \equiv-\mathcal{A} \nabla_{a} T, \\
\gamma_{a b} & \equiv g_{a b}+N_{a} N_{b}, & \mathcal{B}_{a} & \equiv \gamma_{a}{ }^{b} \nabla_{b} T .
\end{aligned}
$$

Similarly, we use the coordinate $R$ to define the corresponding normal vector $S^{a}$, projector $q_{a b}$, length scalar $\mathcal{L}$ and slip vector $b^{a}$

$$
\begin{aligned}
\mathcal{L} & \equiv\left(\gamma^{a b} \nabla_{a} R \nabla_{b} R\right)^{-1 / 2}, & S_{a} & \equiv \mathcal{L} \gamma_{a}{ }^{b} \nabla_{b} R, \\
q_{a b} & \equiv \gamma_{a b}-S_{a} S_{b}, & b_{a} & \equiv q_{a}{ }^{b} \nabla_{b} R .
\end{aligned}
$$

Using the above definitions, the metric $g_{a b}$, written as a line element, reads [23],

$$
\begin{aligned}
\mathrm{d} S^{2}= & -\mathcal{A}^{2} \mathrm{~d} T^{2}+\mathcal{L}^{2}\left(\mathrm{~d} R+\mathcal{L}^{-1} \mathcal{B}^{R} \mathrm{~d} T\right)^{2}+q_{A B}\left(\mathrm{~d} \theta^{A}\right. \\
& \left.+b^{A} \mathrm{~d} R+\mathcal{B}^{A} \mathrm{~d} T\right)\left(\mathrm{d} \theta^{B}+b^{B} \mathrm{~d} R+\mathcal{B}^{B} \mathrm{~d} T\right)
\end{aligned}
$$

On the other hand, the split $g_{a b}=m_{a b}+h_{a b}$ implies

$$
\begin{aligned}
\mathrm{d} s^{2}= & \left(-1+h_{T T}\right) \mathrm{d} T^{2}+2 h_{T R} \mathrm{~d} R \mathrm{~d} T+\left(1+h_{R R}\right) \mathrm{d} R^{2} \\
& +2 \not h_{R A} \mathrm{~d} \theta^{A} \mathrm{~d} R+2 \not h_{T A} \mathrm{~d} \theta^{A} \mathrm{~d} T+\left(R^{2} \not_{A B}\right. \\
& \left.+h_{A B}\right) \mathrm{d} \theta^{A} \mathrm{~d} \theta^{B},
\end{aligned}
$$

where

$$
\begin{array}{ll}
\phi_{A B}=\Omega_{A}{ }^{C} \Omega_{B}{ }^{D} \delta_{C D}, & h_{A B}=\Omega_{A}{ }^{C} \Omega_{B}{ }^{D} h_{\omega_{C} \omega_{D}}, \\
\not h_{R A}=\Omega_{A}{ }^{B} h_{R \omega_{B}}, & h_{T A}=\Omega_{A}{ }^{B} h_{T \omega_{B}} .
\end{array}
$$

Comparing expressions (67) and $(68)$ gives

$$
\begin{aligned}
-1+h_{T T} & =-\mathcal{A}^{2}+\left(\mathcal{B}^{R}\right)^{2}+q_{A B} \mathcal{B}^{A} \mathcal{B}^{B}, \\
1+h_{R R} & =\mathcal{L}^{2}+q_{A B} b^{A} b^{B}, \\
h_{R T} & =q_{A B} b^{A} \mathcal{B}^{B}+\mathcal{L} \mathcal{B}^{R}, \\
q_{A B} & =R^{2}{\phi_{A B}}_{A B} h_{A B}, \\
h_{R A} & =q_{A B} b^{B}, \\
h_{T A} & =q_{A B} \mathcal{B}^{B} .
\end{aligned}
$$

Inverting the matrix encoded in the fifth equation in 770 to write $q^{A B}$ in terms of $\phi_{A B}$ and $\not_{A B}$, the variables of 
the dual foliation formulation can be expressed as,

$$
\begin{aligned}
\mathcal{A} & =\left(\frac{\left(h_{R T}-q^{A B} \not h_{R A} h_{T B}\right)^{2}}{h_{R R}+1-q^{A B} \not h_{R A} h_{R A}}\right. \\
& \left.-\left(h_{T T}-1-q^{A B} h_{T A} h_{T A}\right)\right)^{1 / 2}, \\
\mathcal{B}^{R} & =\frac{h_{R T}-q^{A B} h_{R A} \not h_{T B}}{\sqrt{h_{R R}+1-q^{A B} h_{R A} h_{R A}}}, \\
\mathcal{L} & =\sqrt{h_{R R}+1-q^{A B} \not h_{R A} \not h_{R B}}, \\
b^{A} & =q^{A B} h_{R B}, \\
\mathcal{B}^{A} & =q^{A B} \not h_{T B} .
\end{aligned}
$$

The last expressions can be written in terms of the components of $h_{a b}$ in the flat null frame using that

$$
\begin{aligned}
h_{T T} & =\frac{1}{4}\left(h_{L L}+2 h_{L \underline{L}}+h_{\underline{L L}}\right), \\
h_{R R} & =\frac{1}{4}\left(h_{L L}-2 h_{L \underline{L}}+h_{\underline{L L}}\right), \\
h_{R T} & =\frac{1}{4}\left(h_{L L}-h_{\underline{L L}}\right), \\
h_{R A} & =\frac{1}{2} \Omega_{A}^{B}\left(h_{L \omega_{B}}-h_{\underline{L} \omega_{B}}\right), \\
h_{T A} & =\frac{1}{2} \Omega_{A}^{B}\left(h_{L \omega_{B}}+h_{\underline{L} \omega_{B}}\right) .
\end{aligned}
$$

Recalling that $h_{a b}=\frac{1}{R} H_{a b}$ and taking into account the relation between $\omega_{A}{ }^{a}$ and $S_{A}{ }^{a}$, as given in the expressions (5), gives

$$
\begin{aligned}
h_{L L} & =\frac{1}{R} H_{L L}, & h_{L \underline{L}} & =\frac{1}{R} H_{L \underline{L}}, \\
h_{\underline{L L}} & =\frac{1}{R} H_{\underline{L L}}, & h_{L A} & =\Omega_{A}{ }^{B} H_{L S_{B}}, \\
h_{\underline{L A}} & =\Omega_{A}{ }^{A} H_{\underline{L} S_{A}}, & h_{A B} & =R \Omega_{A}{ }^{C} \Omega_{B}{ }^{D} H_{S_{C} S_{D}} .
\end{aligned}
$$

Now, recall that the radial and angular coordinate lightspeeds in the dual foliation formulation [22, 23] are given by

$$
C_{ \pm}^{R}=-\mathcal{B}^{R} \pm \mathcal{A} \mathcal{L}^{-1}, \quad C_{ \pm}^{A}=-\mathcal{B}^{A} \mp b^{A} \mathcal{A L} \mathcal{L}^{-1}
$$

Thus, using equations (71), (72) and 73) we obtain

$$
\begin{aligned}
& C_{+}^{R} \simeq 1-\frac{H_{L L}}{2 R}, \quad C_{-}^{R} \simeq-1+\frac{H_{\underline{L L}}}{2 R}, \\
& C_{ \pm}^{A}=O^{A}\left(R^{-2}\right) .
\end{aligned}
$$

For completeness of the discussion observe that the variables of the dual foliation formulation are related to the variables of the asymptotic system via

$$
\begin{aligned}
\mathcal{A} & \simeq \pm 1 \mp \frac{H_{\underline{L L}}+2 H_{L \underline{L}}+H_{L L}}{8 R}, \\
\mathcal{L} & \simeq \pm 1 \pm \frac{H_{\underline{L L}}-2 H_{L \underline{L}}+H_{L L}}{8 R}, \\
\mathcal{B}^{R} & \simeq \pm \frac{H_{L L}-H_{\underline{L}}}{4 R}, \\
b^{A} & =O^{A}\left(R^{-2}\right), \\
\mathcal{B}^{A} & =O^{A}\left(R^{-2}\right), \\
q_{A B} & \simeq \Omega_{A}{ }^{A} \Omega_{A}{ }^{B}\left(R^{2} \delta_{A B}+R H_{S_{A} S_{B}}\right) .
\end{aligned}
$$

Using the chain rule one has that

$$
\begin{aligned}
& \partial_{T} C_{+}^{R} \simeq \frac{1}{2 R} \partial_{q} H_{L L}, \\
& \partial_{R} C_{+}^{R} \simeq \frac{1}{2 R^{2}}\left(H_{L L}-\partial_{s} H_{L L}\right)-\frac{1}{2 R} \partial_{q} H_{L L} .
\end{aligned}
$$

The coordinate light-speed condition [22] is that

$$
\partial_{T} C_{+}^{R}=\partial_{\underline{i}} C_{R}^{+}=O\left(R^{-1-\delta}\right)
$$

with $\delta>0$. Consequently, the coordinate light-speed condition is satisfied as long as

$$
H_{L L}+\partial_{s} H_{L L}=O\left(R^{1-\delta}\right), \quad \partial_{q} H_{L L}=O\left(R^{-\delta}\right) .
$$

\section{THE ASYMPTOTIC SYSTEM FOR $\mathcal{T}_{a b}={ }^{\mathscr{I}} \mathcal{T}_{a b}$}

In view of the remarks on made in subsection IVB about free evolution schemes in NR, the following hyperbolic reduction of the Einstein field equations will be considered. Let

$$
{ }^{\mathscr{I}} \mathcal{T}_{a b} \equiv{ }^{\mathcal{C}} \mathcal{T}_{a b}+W_{a b}+\mathcal{W}_{a b}
$$

here $W_{a b}$ and $\mathcal{W}_{a b}$ are symmetric tensors expressed in the flat null frame as in equation (8) where the only nonvanishing components are given by

$$
\begin{aligned}
W_{\underline{L L}} & =C^{c} \stackrel{\circ}{\nabla}_{\underline{L}} g_{\underline{L c}}-\frac{1}{2} C^{c} \stackrel{\circ}{\nabla}_{c} g_{\underline{L L}} \\
\mathcal{W}_{L L} & =\frac{1}{2} C_{L} C_{L}-\frac{\omega}{R} C_{L}, \\
\mathcal{W}_{L S_{A}} & =\frac{1}{2} C_{L} C_{S_{A}}-\frac{\omega}{R} C_{S_{A}}, \\
\mathcal{W}_{S_{A} S_{B}} & =\frac{1}{2} \delta_{A B}\left(\frac{1}{2} C_{L} C_{\underline{L}}-\frac{\omega}{R} C_{\underline{L}}\right) .
\end{aligned}
$$

In these expressions $\omega$ is a positive constant which will play a similar role to the damping parameters $\gamma_{0}, \gamma_{4}$ and $\gamma_{5}$ in [27. First notice that ${ }^{\mathscr{I}} \mathcal{T}_{a b}$ is homogeneous in $C_{a}$ so that $C_{a}=0$ implies ${ }^{\mathscr{I}} \mathcal{T}_{a b}=0$.

The reduced Einstein field equations $\underline{R}_{a b}=0$ imply the following set of wave equations,

$$
g^{c d} \stackrel{\circ}{\nabla}_{d} \stackrel{\circ}{\nabla}_{c} g_{a b}=P_{a b}[g]+Q_{a b}[g]+F_{a b}+2 W_{a b}+2 \mathcal{W}_{a b},
$$


for the metric. Now, we consider $g_{a b}=m_{a b}+h_{a b}$ to compute the corresponding evolution equations for $h_{a b}$ and then its asymptotic system. Here, since we are to discuss the derivation of the corresponding asymptotic system, it is understood, as in subsection IVB, that the metric $m_{a b}$ is used to raise and lower indices of all tensors except for $g_{a b}$ for which one uses equation 50 . Taking these considerations into account, a direct computation using equation 82 renders the following evolution equations for $h_{a b}$,

$$
\begin{aligned}
g^{c d} \stackrel{\circ}{\nabla}_{c} \stackrel{\circ}{\nabla}_{d} h_{a b} & =P_{a b}[h]+Q_{a b}[h]+F_{a b} \\
& +2 W_{a b}+2 \mathcal{W}_{a b}+O_{a b}\left(h(\stackrel{\circ}{\nabla} h)^{2}\right),
\end{aligned}
$$

where it is understood that $F_{a b}, W_{a b}$ and $\mathcal{W}_{a b}$ had been expanded up to order $O_{a b}\left(h(\nabla h)^{2}\right)$. For completeness observe that the GHG constraint reads

$$
m^{a c} \stackrel{\circ}{\nabla}_{c} h_{a b}=\frac{1}{2} m^{a c} \stackrel{\circ}{\nabla}_{b} h_{a c}-F_{b}+O_{b}(h \stackrel{\circ}{\nabla} h),
$$

where $O_{b}(h \stackrel{\circ}{\nabla} h)$ is linear in $\stackrel{\circ}{\nabla}_{a} h_{b c}$ with coefficients depending on $h_{a b}$ which vanish for $h_{a b}=0$.

Recall that the asymptotic system is extracted from the terms corresponding to the leading order of $\underline{R}_{a b}=0$, expressed in the asymptotic variables $H_{a b}$ and coordinates $(q, s)$, which in the harmonic case discussed in subsection IV B is $O\left(R^{-2}\right)$. Thus one can see that setting $F^{a} \simeq \nabla_{b} F^{a}=O\left(R^{-3}\right)$ ensures that the gauge source functions will not contribute to the asymptotic system. Furthermore, with such a choice of $F^{a}$, the GHG asymptotic constraint equations, i.e. the asymptotic expressions for $C^{a}$, coincide with the harmonic case since the latter are obtained from the asymptotic expression for $\Gamma^{a}$ whose leading order is $O\left(R^{-1}\right)$. On the other hand, observe that $W_{a b}$ and $\mathcal{W}_{a b}$ do contribute to the asymptotic system since their components are either quadratic in $C_{a}$ or proportional to $R^{-1} C_{a}$.

Computing the asymptotic system for equation (82) one obtains

$$
\left(2 \stackrel{\circ}{\nabla}_{s}-H_{L L} \stackrel{\circ}{\nabla}_{q}\right) \stackrel{\circ}{\nabla}_{q} H_{a b}=T_{a b},
$$

where $T_{a b}$ is expressed in the flat null frame as (8) where the only non-vanishing components are

$$
\begin{aligned}
T_{L L} & =\left(-2 \omega+\partial_{q} H_{L L}\right) \partial_{q} H_{L L}, \\
T_{L S_{A}} & =\left(-2 \omega+\partial_{q} H_{L L}\right) \partial_{q} H_{L S_{A}}, \\
T_{S_{A} S_{B}} & =\delta_{A B}\left(-\omega+\frac{1}{2} \partial_{q} H_{L L}\right)\left(\partial_{q} H^{\varnothing}\right) \\
T_{\underline{L L}} & =-4\left(\partial_{q} H^{+}\right)^{2}-4\left(\partial_{q} H^{\times}\right)^{2}
\end{aligned}
$$

On the other hand, as explained above, the asymptotic GHG constraint condition is identical to equation 62 for our choice of $F^{a}$. A first observation is that contracting with $\underline{L}^{a} \underline{L}^{b}$ equation (64) is of course recovered. The term $W_{a b}$ was included precisely for this purpose. The advantage of this approach is that the above succinct expression for $T_{L L}$ is obtained directly from the evolution equations without assuming that $C^{a}=0$. As it will be discussed in greater detail in the next subsection, the parameter $\omega$ in the term $\mathcal{W}_{a b}$ was included so that the evolution equations ensure the small constraint violations are damped away along the integral curves of then vector field $2 \partial_{s}-H_{L L} \partial_{q}$ while the terms quadratic in $C_{a}$ in with $\mathcal{W}_{a b}$ are introduced to be able to commute $2 \partial_{s}-H_{L L} \partial_{q}$ and $\partial_{q}$ when necessary. A detailed discussion on how to exploit these constraint additions in view of a future numerical implementation using the dual foliation formalism is the content of the next subsection.

\section{A. Analysis of the asymptotic system}

A direct computation then shows that the asymptotic system 85 can be expressed as

$$
\begin{aligned}
& \left(\partial_{s}-\frac{H_{L L}}{2} \partial_{q}\right) \partial_{q} H_{\mathcal{U}}=-\omega \partial_{q} H_{\mathcal{U}}+\frac{1}{2} \partial_{q} H_{\mathcal{U}} \partial_{q} H_{L L}, \\
& \left(\partial_{s}-\frac{H_{L L}}{2} \partial_{q}\right) \partial_{q} H_{\mathcal{V}}=0, \\
& \left(\partial_{s}-\frac{H_{L L}}{2} \partial_{q}\right) \partial_{q} H_{\underline{L L}}=-2\left(\partial_{q} H^{+}\right)^{2}-2\left(\partial_{q} H^{\times}\right)^{2},
\end{aligned}
$$

where $\mathcal{V} \in\left\{L \underline{L}, \underline{L} S_{A}, \times,+\right\}$ and $\mathcal{U} \in\left\{L L, L S_{A}, \varnothing\right\}$.

Observe the asymptotic equations for the components of $H_{a b}$, split in three classes whereas in the asymptotic system of subsection IV B they split only in two. The components of $H_{a b}$ satisfying equation $87 \mathrm{~b}$ will be referred as the "the good" components, on the other hand, $H_{\underline{L}}$ will be referred as "the bad" component and finally, those satisfying equation (87a) as the "the ugly" components. Observe that the asymptotic system (87) does not follow the hierarchical structure introduced in [25. In particular, the equation for $H_{L L}$ does not lie on the bottom level of the hierarchy and its equation contains quadratic non-linearities of $H_{L L}$ itself. Nevertheless we will see in what follows that one can still integrate these equations and obtain, under suitable assumptions on the initial data, bounded solutions for $H_{\mathcal{U}}$ and moreover, that $\partial_{q} H_{\mathcal{U}} \sim O\left(R^{-\omega}\right)$.

For completeness, observe that, with the current decay assumption on the gauge source functions $F^{a}$, the asymptotic GHG constraint equations are identical to those given in equations 63 and can be compactly written as

$$
C_{\mathcal{U}}=0
$$

where $C_{\mathcal{U}} \equiv \partial_{q} H_{\mathcal{U}}$. Nevertheless, recall, that the conditions encoded in (88) are not expected to be fulfilled in a free evolution numerical implementation as one expects small violations to the GHG constraints. Consequently, the following analysis will be done without assuming that equation 88 is satisfied.

Solution for the uglies: Observe that the equations encoded in 87a are the asymptotic equations for the components of the metric perturbation associated with the GHG constraints. In other words, equation (87a) can 
be read as the following equation for the GHG constraints

$$
\left(\partial_{s}-\frac{1}{2} H_{L L} \partial_{q}\right) C_{\mathcal{U}}=-\omega C_{\mathcal{U}}+\frac{1}{2} C_{\mathcal{U}} C_{L L} .
$$

Let $\gamma=\left(s(\lambda), q(\lambda), \theta^{A}(\lambda)\right)$ be a curve passing through the point $\gamma_{\star}=\left(s_{\star}, q_{\star}, \theta_{\star}^{A}\right)$, where $s_{\star}=s\left(\lambda_{\star}\right), q_{\star}=q\left(\lambda_{\star}\right)$ and $\theta_{\star}^{A}=\theta^{A}\left(\lambda_{\star}\right)$, satisfying

$$
\frac{\mathrm{d} s}{\mathrm{~d} \lambda}=1, \quad \frac{\mathrm{d} q}{\mathrm{~d} \lambda}=-\frac{1}{2} H_{L L},
$$

and $\theta^{A}(\lambda)=\theta_{\star}^{A}$. Observe that integrating the first equation in $(90)$ and setting $\lambda_{\star}=s_{\star}$ one has $s=\lambda$. Thus, one can use $s$ to parameterize the curve as $\gamma=\left(s, q(s), \theta_{\star}^{A}\right)$. Then, considering equation 89 along $\gamma$ one has

$$
\frac{\mathrm{d} C_{\mathcal{U}}(\gamma)}{\mathrm{d} s}=-\omega C_{\mathcal{U}}(\gamma)+\frac{1}{2} C_{\mathcal{U}}(\gamma) C_{L L}(\gamma)
$$

Solving equation for $\mathcal{U}=L L$ one obtains,

$$
C_{L L}(\gamma)=\frac{2 \omega}{1-e^{\omega\left(p+2 A_{\star}\right)}}
$$

where $A_{\star}$ is an integration constant. Using that $\omega>0$ and denoting the initial datum $C_{L L}\left(s_{\star}, q_{\star}\right)$ as $C_{L L}\left(\gamma_{\star}\right)$ then the solution can be written as

$$
C_{L L}(\gamma)=\frac{2 C_{L L}\left(\gamma_{\star}\right) \omega e^{\omega s_{\star}}}{C_{L L}\left(\gamma_{\star}\right)\left(e^{\omega s_{\star}}-e^{\omega s}\right)+2 \omega e^{\omega s}} .
$$

This equation is dangerous since one could have blow up at finite value of $s$. Nevertheless, assuming that $C_{L L}\left(\gamma_{\star}\right)<2 \omega$ the denominator in expression is positive, and then $C_{L L}(\gamma)$ is bounded. Moreover, one has that

$$
\lim _{s \rightarrow \infty} C_{L L}(\gamma)=0
$$

Now, we can use expression 93 to solve equation 89 for $C_{\mathcal{U}}$ with $\mathcal{U} \in\left\{L S_{A}, \varnothing\right\}$. A direct calculation yields,

$$
C_{\mathcal{U}}(\gamma)=\frac{2 \mathcal{C}_{\mathcal{U}}\left(\gamma_{\star}\right) \omega e^{\omega s_{\star}}}{\mathcal{C}_{L L}\left(\gamma_{\star}\right)\left(e^{\omega s_{\star}}-e^{\omega s}\right)+2 \omega e^{\omega s}} .
$$

Again observe that

$$
\lim _{s \rightarrow \infty} C_{\mathcal{U}}(\gamma)=0
$$

Consequently, recalling that $C_{\mathcal{U}} \equiv \partial_{q} H_{\mathcal{U}}$, then, one concludes that, $\partial_{q} H_{\mathcal{U}}(\gamma)$ is determined by equations (93) and (94). Now, to solve for $H_{\mathcal{U}}$ observe that

$$
\begin{array}{r}
\partial_{q}\left(2 \partial_{s}-H_{L L} \partial_{q}\right) H_{\mathcal{U}}=\left(2 \partial_{s}-H_{L L} \partial_{q}\right) \partial_{q} H_{\mathcal{U}} \\
-\partial_{q} H_{L L} \partial_{q} H_{\mathcal{U}}
\end{array}
$$

Using the latter identity with equation $87 \mathrm{a}$ we get

$$
\partial_{q}\left[\left(2 \partial_{s}-H_{L L} \partial_{q}\right) H_{\mathcal{U}}\right]=-2 \omega \partial_{q} H_{\mathcal{U}}
$$

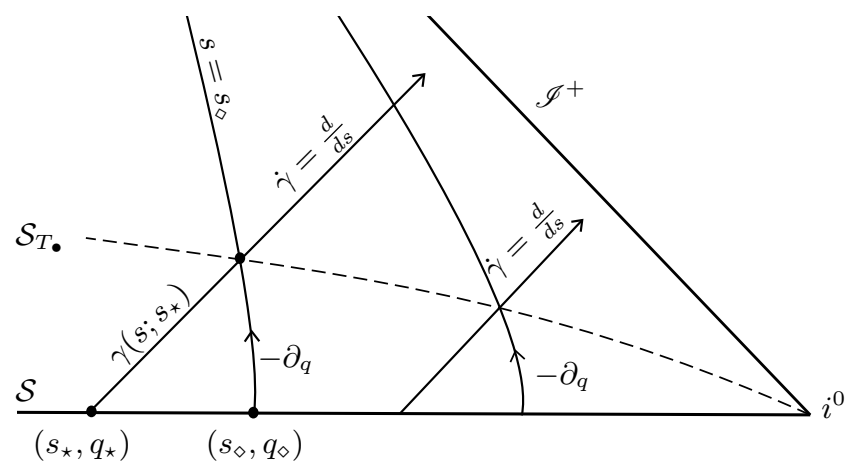

FIG. 1: Schematic depiction of the integration of the components of $H_{a b}$ associated with the constraints. The angular coordinates $\theta_{\star}^{A}$ have been suppressed to simplify the notation in this figure and this caption. Given a point $\left(s_{\star}, q_{\star}\right)$ on the initial hypersurface $\mathcal{S}$, the characteristic curve that passes through that point is denoted as $\gamma\left(s ; s_{\star}\right)$. Observe that varying $s_{\star}$, then one obtains a congruence of curves parameterized by $s_{\star}$. The diagram shows the hypersurface $S_{T_{\bullet}}$ determined by the condition $T=T_{\bullet}$ where, in turn, $T_{\bullet}$, is determined by the intersection of the curve with constant $s=s_{\diamond}$ and the characteristic $\gamma\left(s ; s_{\star}\right)$ as shown in the diagram. The integral curves of $\partial_{q}$ are the curves of constant $s$ along which the first integration is performed. Then, the second integration is performed along the characteristic curves $\gamma\left(s ; s_{\star}\right)$

Consequently, integrating from $q_{0}$ to $q$ we have

$$
\left(2 \partial_{s}-H_{L L} \partial_{q}\right) H_{\mathcal{U}}+2 \omega H_{\mathcal{U}}=\mathcal{G}_{\mathcal{U}}(s),
$$

where

$$
\left.\mathcal{G}_{\mathcal{U}}(s) \equiv\left(\left(2 \partial_{s}-H_{L L} \partial_{q}\right) H_{\mathcal{U}}+2 \omega H_{\mathcal{U}}\right)\right|_{\mathcal{S}},
$$

and $\mathcal{S}$ is the hypersurface determined by $T=0$. Observe that if $p \in \mathcal{S}$, then $p$ has coordinates $\left(s, q_{0}(s), \theta^{A}\right)$ where $q_{0}(s)=e^{s}$.

Now, consider the curve $\gamma$ intersecting the hypersurface $\mathcal{S}$ at the point $p_{\star}$ with coordinates $\left(s_{\star}, q_{\star}, \theta_{\star}^{A}\right)$, where $q_{\star}=e^{s_{\star}}$. Proceeding similarly as in equation (91) one can rewrite equation $(98)$ as

$$
\frac{\mathrm{d} H_{\mathcal{U}}(\gamma)}{\mathrm{d} s}+\omega H_{\mathcal{U}}(\gamma)=\frac{1}{2} \mathcal{G}_{\mathcal{U}}(s)
$$

The latter equation can be solved to yield

$$
H_{\mathcal{U}}(\gamma)=e^{-\omega s}\left(e^{\omega s_{\star}} H_{\mathcal{U}}\left(\gamma_{\star}\right)-\frac{1}{2} \int_{s_{\star}}^{s} e^{\omega \bar{s}} \mathcal{G}_{\mathcal{U}}(\bar{s}) \mathrm{d} \bar{s}\right)
$$

Thus, $H_{\mathcal{U}}(\gamma)$ and $\partial_{q} H_{\mathcal{U}}(\gamma)$ are determined by data on $\mathcal{S}$ via equations (101), (93) and (94). Additionally, observe that, from equation (98) one has

$$
\partial_{s} H_{\mathcal{U}}=\frac{1}{2} H_{L L}(\gamma) \partial_{q} H_{\mathcal{U}}(\gamma)-\omega H_{\mathcal{U}}+\frac{1}{2} \mathcal{G}_{\mathcal{U}}(s) .
$$

Therefore, $\partial_{s} H_{\mathcal{U}}(\gamma)$ is determined by $H_{\mathcal{U}}(\gamma), \partial_{q} H_{\mathcal{U}}(\gamma)$ and $\mathcal{G}_{\mathcal{U}}(s)$. Finally, recalling that $s=\ln R$, then, if the 
initial data on $\mathcal{S}$ is such that

$$
\begin{array}{rlrl}
\left.H_{\mathcal{U}}\right|_{\mathcal{S}} & \sim O(1), & & \sup \left(\left.\partial_{q} H_{L L}\right|_{\mathcal{S}}\right)<2 \omega . \\
\left.\partial_{q} H_{\mathcal{U}}\right|_{\mathcal{S}} \sim O\left(R^{-1}\right), & & \left.\partial_{s} H_{\mathcal{U}}\right|_{\mathcal{S}} \sim O\left(R^{-1}\right),
\end{array}
$$

Then $\mathcal{G}_{\mathcal{U}}(s) \simeq O(1)$ and, consequently, using equations (101), 93) and (94) one concludes that

$$
\begin{aligned}
\partial_{q} H_{\mathcal{U}}(\gamma) & \sim O\left(R^{-\omega}\right), \quad \partial_{s} H_{\mathcal{U}}(\gamma) \sim O(1), \\
H_{\mathcal{U}}(\gamma) & \sim O(1) .
\end{aligned}
$$

Observe that, since $\partial_{R}=\frac{1}{R} \partial_{s}$ then $\partial_{s} H_{\mathcal{U}}(\gamma) \sim O(1)$ implies that $\partial_{R} H_{\mathcal{U}}(\gamma) \sim O\left(R^{-1}\right)$. The latter decay rates imply that the coordinate light-speed condition, as expressed in equation $(79)$, is satisfied if one sets $\omega \geq \delta$.

Solution for the goods: Notice that in contrast with the case of equation 87a), for equation 87b the fields $\partial_{q} H_{\mathcal{V}}$ are not associated to the constraints. Nevertheless, along the curve $\gamma$ equation $87 \mathrm{~b}$ simply reads

$$
\frac{\mathrm{d}}{\mathrm{d} s} \partial_{q} H_{\mathcal{V}}(\gamma)=0
$$

Thus

$$
\partial_{q} H_{\mathcal{V}}(\gamma)=\partial_{q} H_{\mathcal{V}}\left(\gamma_{\star}\right)
$$

Now, integrating the second equation in 90 one has that

$$
q=q_{\star}-\frac{1}{2} \int_{s_{\star}}^{s} H_{L L}(\bar{s}) \mathrm{d} \bar{s} .
$$

Observe that $\partial_{q} H_{\mathcal{V}}\left(\gamma_{\star}\right)=\partial_{q} H_{\mathcal{V}}\left(s_{\star}, q_{\star}\right)$ does not depend on $s$. Using $q_{\star}$ as a new coordinate. Namely, considering a coordinate system $(s, u)$ where $u=q+\frac{1}{2} \int_{s_{\star}}^{s} H_{L L}(\bar{s}) \mathrm{d} \bar{s}$ then $\partial_{q} H_{\mathcal{V}}\left(\gamma_{\star}\right)$ is only a function of $u$. Moreover, since $\partial_{q} H_{\mathcal{V}}=\partial_{u} H_{\mathcal{V}}$ then, integrating in $u$ one has

$$
H_{\mathcal{V}}(\gamma)=\int_{u_{\star}}^{u} \partial_{q} H_{\mathcal{V}}(\bar{u}) \mathrm{d} \bar{u}
$$

Thus, $H_{\mathcal{V}}(\gamma)$ does not depend on $s$, consequently, $H_{\mathcal{V}}(\gamma) \sim O(1)$.

Solution for the bad: To integrate equation $87 \mathrm{c}$ observe that along the curve $\gamma$ one has

$$
\frac{\mathrm{d}}{\mathrm{d} s} \partial_{q} H_{\underline{L L}}(\gamma)=-2\left[\left(\partial_{q} H^{+}\right)^{2}-2\left(\partial_{q} H^{\times}\right)^{2}\right] .
$$

Using the previous results obtained for $H_{\mathcal{V}}$, one has that $\partial_{q} H^{+}$and $\partial_{q} H^{\times}$do not depend on $s$. Thus, integrating in $s$, one obtains

$$
\partial_{q} H_{\underline{L L}}(\gamma)=-2\left[\left(\partial_{q} H^{+}\left(\gamma_{\star}\right)\right)^{2}+\left(\partial_{q} H^{\times}\left(\gamma_{\star}\right)\right)^{2}\right] s .
$$

Then, using the coordinates $(s, u)$ and that $\partial_{q}=\partial_{u}$, one has that

$$
H_{\underline{L L}}(\gamma)=-2 s \int_{u_{\star}}^{u}\left(\left(\partial_{q} H^{+}\left(\gamma_{\star}\right)\right)^{2}+\left(\partial_{q} H^{\times}\left(\gamma_{\star}\right)\right)^{2}\right) \mathrm{d} \bar{u} .
$$

Observe that the integration of the asymptotic equation for the goods and the bad is analogous to that of the model equation (17).

\section{B. The eikonal equation}

In this subsection the asymptotic form of the eikonal equation and its relation to the characteristics of the asymptotic system 85 are discussed. As emphasized before, when dealing with asymptotic computations we will use the Minkowski metric $m_{a b}$ to raise and lower indices in all tensors except for $g_{a b}$ in which case we use equation 50.

The eikonal equation and its asymptotic system: The vectors $L^{a}$ and $\underline{L}^{a}$ are null vectors in Minkowski spacetime, namely $m^{a b} L_{a} L_{b}=m^{a b} \underline{L}_{a} \underline{L}_{b}=0$. In what follows, the asymptotic form of vectors $\xi^{a}$ which are null respect to the perturbed metric $g_{a b}$ will be discussed.

We start by recalling that the eikonal equation reads

$$
g^{a b} \nabla_{a} u \nabla_{b} u=0,
$$

and that if $\xi^{a}=g^{a b} \nabla_{b} u$, then eikonal equation simply states that $\xi^{a}$ is null respect to $g_{a b}$. Now, let

$$
\xi^{a}=\stackrel{\circ}{\xi}^{a}+\frac{1}{R} \check{\xi}^{a},
$$

where

$$
m_{a b} \stackrel{\circ}{\xi}^{a} \dot{\xi}^{b}=0 .
$$

Using this notation one has that

$$
\begin{aligned}
g_{a b} \xi^{a} \xi^{b} & =\frac{H_{a b} \stackrel{\circ}{\xi}^{a} \stackrel{\circ}{\xi}^{b}}{R}+\frac{2 m_{a b} \stackrel{\circ}{\xi}^{a} \check{\xi}^{b}}{R}+\frac{2 H_{a b} \stackrel{\circ}{\xi}^{a} \check{\xi}^{b}}{R^{2}} \\
& +\frac{m_{a b} \check{\xi}^{a} \check{\xi}^{b}}{R^{2}}+\frac{H_{a b} \check{\xi}^{a} \check{\xi}^{b}}{R^{3}} .
\end{aligned}
$$

Thus, $\xi^{a}$ is a null vector respect to $g_{a b}$ to leading order $R^{-1}$, if the following condition is satisfied

$$
H_{a b} \stackrel{\circ}{\xi}^{a} \stackrel{\circ}{ }^{b}+2 m_{a b} \stackrel{\circ}{\xi}^{a} \check{\xi}^{b}=0 .
$$

Naturally, one can algebraically impose that $g_{a b} \xi^{a} \xi^{b}=0$ also to order $R^{-2}$ and $R^{-3}$. Nevertheless, consistent with the discussion of the asymptotic system, one is interested only in the leading order. In the following, it will be shown that, if equation (116) is satisfied on the initial hypersurface $\mathcal{S}$ then $\xi^{a}$ will be, to leading order, null respect to $g_{a b}$ in $\mathcal{D}(\mathcal{S}) \subseteq \mathcal{M}$ provided that the coordinate light-speed condition of subsection IV C is satisfied. The condition imposed by the eikonal equation $(112$ can be formulated as an initial value problem as follows

$$
\nabla_{c}\left(g_{a b} \xi^{a} \xi^{b}\right)=0,\left.\quad\left(g_{a b} \xi^{a} \xi^{b}\right)\right|_{\mathcal{S}}=0 .
$$

The initial condition $\left.\left(g_{a b} \xi^{a} \xi^{b}\right)\right|_{\mathcal{S}}=0$ to leading order $R^{-1}$ corresponds to equation (116) where the fields are restricted to the initial hypersurface $\mathcal{S}$.

Now observe that the condition $\xi^{a}=g^{a b} \nabla_{b} u$ is equivalent to the integrability condition $\nabla_{[a} \xi_{b]}=0$. Using this integrability condition, the first equation in 117 reduces to the geodesic equation

$$
\xi^{b} \nabla_{b} \xi^{a}=0
$$


Using the integrability condition $\stackrel{\circ}{\nabla}_{[a} \stackrel{\circ}{\xi}_{b]}=0$ and proceeding in analogous way with equation (114) one has,

$$
\stackrel{\circ}{\xi}^{b} \stackrel{\circ}{\nabla}_{b} \stackrel{\circ}{ }^{a}=0 .
$$

Notice that the geodesic equation 118 can be expressed more explicitly, using the covariant derivative $\stackrel{\circ}{\nabla}$, as

$$
2 \xi^{a} \stackrel{\circ}{\nabla}_{a} \xi^{c}+\xi^{a} \xi^{b}(g)^{c d}\left(\stackrel{\circ}{\nabla}_{a} g_{b d}+\stackrel{\circ}{\nabla}_{b} g_{a d}-\stackrel{\circ}{\nabla}_{d} g_{a b}\right)=0 .
$$

Using equations (49, (54), 113, 118) and (119) one obtains

$$
\begin{array}{r}
\xi^{a} \nabla_{a} \xi^{c} \simeq \frac{1}{R}\left(2 \stackrel{\circ}{\xi}^{a} \stackrel{\circ}{\nabla}_{a} \check{\xi}^{c}+2 \check{\xi}^{a} \stackrel{\circ}{\nabla}_{a} \stackrel{\circ}{\xi}^{c}+m^{c d}\left(\stackrel{\circ}{\xi}^{a} \dot{\circ}^{b} \stackrel{\circ}{\nabla}_{a} H_{b d}\right.\right. \\
\left.\left.+\dot{\circ}^{a} \stackrel{\circ}{\xi}^{b} \stackrel{\circ}{\nabla}_{b} H_{a d}-\stackrel{\circ}{\xi}^{a} \stackrel{\circ}{\xi}^{b} \stackrel{\circ}{\nabla}_{d} H_{a b}\right)\right)
\end{array}
$$

Thus, similarly as it was done for the Einstein field equations, one says that the asymptotic system for equation (118) is encoded in the leading order of the above expansion. Recall that $\dot{\xi}^{a}$ represents any null vector with respect to $m_{a b}$. Choosing $\dot{\circ}^{a}=L^{a}$ and contracting with the flat null frame one obtains that

$$
\begin{aligned}
\underline{\hat{L}}_{c} \xi^{a} \nabla_{a} \xi^{c} & \simeq \frac{2}{R} \stackrel{\circ}{\nabla}_{q} H_{L L}, \\
\hat{L}_{c} \xi^{a} \nabla_{a} \xi^{c} & =O\left(R^{-2}\right), \\
\hat{S}^{A}{ }_{c} \xi^{a} \nabla_{a} \xi^{c} & =O\left(R^{-2}\right) .
\end{aligned}
$$

Recalling from subsection $\mathrm{VA}$ that, along the characteristic curve $\gamma$, one has $\nabla_{q} H_{\mathcal{U}} \simeq O\left(R^{-\omega}\right)$ we observe that setting $\omega \geq 1$ ensures that equation 120 is satisfied to leading order $R^{-1}$.

Therefore, exploiting the discussion of subsection $\mathrm{VA}$ and setting $\omega \geq 1$, one can conclude that

$$
\left.\left(g_{a b} \xi^{a} \xi^{b}\right)\right|_{\mathcal{S}}=0
$$

implies that, to leading order,

$$
g_{a b} \xi^{a} \xi^{b}=0, \quad \text { in } \quad \mathcal{D}(\mathcal{S}) \subseteq \mathcal{M}
$$

Additionally, observe that for $\stackrel{\circ}{\xi}^{a}=L^{a}$, using equation (116), condition (123) reduces to

$$
\left.\left(\hat{L}_{a} \check{\xi}^{a}\right)\right|_{\mathcal{S}}=-\left.\frac{1}{2} H_{L L}\right|_{\mathcal{S}}
$$

By the argument above, to leading order, one has $g_{a b} \xi^{a} \xi^{b}=0$ in $\mathcal{D}(\mathcal{S}) \subseteq \mathcal{M}$. Using equation (116), this is equivalent to

$$
\hat{L}_{a} \check{\xi}^{a}=-\frac{1}{2} H_{L L}, \quad \text { in } \quad \mathcal{D}(\mathcal{S}) \subseteq \mathcal{M} .
$$

The characteristics of the asymptotic system and null geodesics: We now show that the characteristics of the asymptotic system (85) correspond, to leading order, to null geodesics of the perturbed spacetime $\left(g_{a b}, \mathcal{M}\right)$. Observe that, using equations (5) and (54) one has that

$$
\left(\partial_{q}\right)^{a}=-\frac{1}{2}\left(L^{a}+\underline{L}^{a}\right), \quad\left(\partial_{s}\right)^{a}=R L^{a} .
$$

We define

$$
\chi^{a}=\frac{1}{R}\left(\left(\partial_{s}\right)^{a}-\frac{1}{2} H_{L L}\left(\partial_{q}\right)^{a}\right),
$$

and observe that $\chi^{a}$ is a tangent to the curve $\gamma$. Furthermore, notice that $\chi^{a}$ can be expressed as

$$
\chi^{a}=L^{a}+\frac{1}{4 R} H_{L L}\left(L^{a}+\underline{L}^{a}\right) .
$$

Now, consistent with equation (113), consider the split $\chi^{a}=\dot{\chi}^{a}+\frac{1}{R} \check{\chi}^{a}$ with $\dot{\circ}^{a}=L^{a}$ and

$$
\check{\chi}^{a}=\frac{1}{4} H_{L L}\left(L^{a}+\underline{L}^{a}\right) .
$$

Then, one readily observes that

$$
\hat{L}_{a} \check{\chi}^{a}=-\frac{1}{2} H_{L L}
$$

Consequently, using the results of subsection $\mathrm{VB}$, one concludes that the vector $\chi^{a}$ representing the tangent vector to the characteristics of the asymptotic system 85, is, to leading order, a null vector in the perturbed spacetime $\left(g_{a b}, \mathcal{M}\right)$.

\section{THE ASYMPTOTIC SYSTEM FOR FIRST ORDER GHG WITH CONSTRAINT DAMPING}

In this section, the first order GHG Einstein evolution equations with the constraint additions introduced in section $\mathrm{V}$ are presented and its asymptotic system is derived in analogous way as that of the model equation (17). In subsection VIA the general evolution equations are given, and, since this is not an asymptotic computation, the usual conventions for raising and lowering indices using $g_{a b}$ is employed. In contrast, in subsection VIB the corresponding asymptotic system is derived, and, consequently, the indices are handled in concordance with the asymptotic calculations of section IVB.

\section{A. First order GHG}

Consider the reduced Ricci operator as given in equation (41), where $\mathcal{T}_{a b}$ denotes a generic constraint addition. Then, using the foregoing conventions by expressing the derivatives in terms of $\stackrel{\circ}{\nabla}^{\circ}$, the GHG evolution equations 22], in abstract index notation, read

$$
\begin{aligned}
& N^{c} \stackrel{\circ}{\nabla}_{c} g_{a b}=S_{a b}^{(g)} \\
& N^{f} \gamma_{a}{ }^{d} \stackrel{\circ}{\nabla}_{f} \phi_{d b c}=-\gamma_{a}{ }^{d} \stackrel{\circ}{\nabla}_{d} \pi_{b c}+\gamma_{2} \gamma_{a}{ }^{d} \stackrel{\circ}{\nabla}_{d} g_{b c}+S_{a b c}^{(\phi)} \\
& N^{c} \nabla_{c}^{\circ} \pi_{a b}=\gamma^{c d} \stackrel{\circ}{\nabla}_{c} \phi_{d a b}+S_{a b}^{(\pi)}
\end{aligned}
$$


where

$S_{a b}^{(g)}=-\pi_{a b}$

$S_{a b c}^{(\phi)}=-\gamma_{2} \phi_{a b c}+\frac{1}{2} N^{d} N^{h} \pi_{b c} \phi_{a d h}+\gamma^{f h} N^{d} \phi_{f b c} \phi_{a d h}$

$S_{a b}^{(\pi)}=-2 \nabla_{(a} F_{b)}-2 \Gamma_{a c h} \Gamma_{b d f} g^{c d} g^{h f}-\frac{1}{2} N^{c} N^{d} \pi_{a b} \pi_{c d}$

$-2 g^{c d} \pi_{c a} \pi_{d b}-N^{d} \pi_{d f} \gamma^{c f} \phi_{c a b}+2 g^{c d} g^{h f} \phi_{f d b} \phi_{h c a}+2 \mathcal{T}_{a b}$

and

$$
\Gamma_{a b c}=N_{(c} \pi_{b) a}-\frac{1}{2} N_{a} \pi_{b c}+\phi_{(c|a| b)}-\frac{1}{2} \phi_{a b c} .
$$

These evolution equations were written respect to normal derivatives to ease the subsequent discussion. Nevertheless, observe that time derivatives and normal derivatives are related via

$$
\stackrel{\circ}{\nabla}_{T}=\mathcal{A} N^{a} \stackrel{\circ}{\nabla}_{a}+\mathcal{B}^{a} \stackrel{\circ}{\nabla}_{a}
$$

See 28] for a discussion of these equations and the appended mathematica notebooks for a detailed derivation.

Notice that in the notation of 22 the formulation parameters $\gamma_{0}, \gamma_{3}$ have been set to zero and, ignoring the potential parameters in the constraint addition term $\mathcal{T}_{a b}$, $\gamma_{2}$ is the only parameter that has been left unspecified. The evolution system 132 is subject, as in the discussion of section $\mathrm{V}$, to the GHG constraints

$$
C^{a} \equiv \Gamma^{a}+F^{a}=0,
$$

and, additionally, subject to the reduction constraints

$$
\mathcal{C}_{a b c} \equiv \gamma_{a}{ }^{d} \nabla_{d} g_{b c}-\phi_{a b c}=0 .
$$

\section{B. Evolution equations for the perturbation}

To derive the asymptotic system we proceed as in previous sections and set

$$
g_{a b}=m_{a b}+h_{a b} .
$$

Following the discussion of the asymptotic system in subsection IV B, once the equations for the perturbations are obtained, as for example in equation $(52)$, to derive the asymptotic system, the metric $g_{a b}$ is regarded just as a symmetric tensor and the indices are raised and lowered using $m_{a b}$. The latter implies that one has to be very careful with the canonical position of indices before the expansion. In particular recall that for the inverse metric one has

$$
g^{a b}=m^{a b}-h^{a b}+O^{a b}\left(h^{2}\right)
$$

where the indices in the right hand side of the above expansion are moved using $m$, in other words, $h^{a b}=$ $m^{a c} m^{d b} h_{c d}$. To simplify the notation and avoid lengthier expressions, let $\doteq$ denote equality up to terms of order $O\left(h^{2}, h \stackrel{\circ}{\nabla} h,\left(\stackrel{\circ}{\nabla}^{2} h\right)^{2}\right)$ so that one writes,

$$
g^{a b} \doteq m^{a b}-h^{a b} .
$$

The latter observations imply the following expansions for the normal and projector

$$
\begin{aligned}
N_{a} & \doteq \stackrel{\circ}{N}_{a}+\check{N}_{a}, \\
\gamma_{a b} & \doteq \check{\gamma}_{a b}+\check{\gamma}_{a b}, \\
N^{a} \equiv\left(g^{-1}\right)^{a b} N_{b} & \doteq \stackrel{\circ}{N}^{a}+\left(\check{N}^{a}-\stackrel{\circ}{b}_{b} h^{a b}\right), \\
\gamma_{a}{ }^{b} \equiv\left(g^{-1}\right)^{c b} \gamma_{a c} & \doteq \check{\gamma}_{a}{ }^{b}+\left(-h^{c b} \stackrel{\gamma}{\alpha c}_{a c}+\check{\gamma}_{a}{ }^{b}\right), \\
\gamma^{a b} \equiv\left(g^{-1}\right)^{a c}\left(g^{-1}\right)^{b d} \gamma_{c d} & \doteq \check{\gamma}^{a b}+\left(\check{\gamma}^{a b}-h^{c a} \dot{\gamma}_{c}{ }^{b}-h^{d b} \dot{\gamma}_{d}{ }_{d}\right)
\end{aligned}
$$

where,

$$
\begin{array}{lrl}
\stackrel{\circ}{\gamma}_{a b} \equiv m_{a b}+\stackrel{\circ}{N}_{a} \stackrel{\circ}{N}_{b} & \check{\gamma}_{a b} \equiv 2 \stackrel{\circ}{N}_{(a} \check{N}_{b)}+h_{a b} \\
\stackrel{\circ}{N}_{a} \equiv-\frac{1}{2}\left(L_{a}+\underline{L}_{a}\right) & \check{N}_{a} \equiv-\frac{1}{2} \stackrel{\circ}{N}_{a} \stackrel{\circ}{ }^{b} \stackrel{\circ}{N}^{c} h_{b c}
\end{array}
$$

and all the indices in the right-hand side of equation (141) were moved using $m_{a b}$. Since $\nabla_{c}^{\circ} m_{a b}=0$ then, one has that the background values for $\pi_{a b}, \phi_{a b c}$ and $\Gamma_{a b c}$ vanish, namely,

$$
\stackrel{\circ}{\pi}_{a b}=\stackrel{\circ}{\phi}_{a b c}=\stackrel{\circ}{\Gamma}_{a b c}=0 .
$$

To simplify the notation we will denote the corresponding perturbations to the latter quantities without adding to each of these fields. Thus, hereafter, $\pi_{a b}, \phi_{a b c}$ and $\Gamma_{a b c}$ will represent the associated perturbations. A straightforward computation renders the following evolution equations

$$
\begin{aligned}
N^{a} \stackrel{\circ}{\nabla}_{a} h_{b c} & \doteq-\pi_{b c}, \\
N^{a} \gamma_{f}^{b} \stackrel{\circ}{\nabla}_{a} \phi_{b c d} & \doteq \frac{1}{2} N^{a} N^{b} \pi_{c d} \phi_{f a b}+N^{a} \gamma^{b h} \phi_{b c d} \phi_{f a h} \\
& +\gamma_{2}\left(-\phi_{f c d}+\gamma_{f}^{a} \stackrel{\circ}{\nabla}_{a} h_{c d}\right)-\gamma_{f}^{a} \stackrel{\circ}{\nabla}_{a} \pi_{c d}, \\
N^{c} \stackrel{\circ}{\nabla}_{c} \pi_{a b} & \doteq-2 m^{c d} m^{h f} \Gamma_{a c h} \Gamma_{b d f}-\frac{1}{2} N^{c} N^{d} \pi_{a b} \pi_{c d} \\
& -2 m^{c d} \pi_{c a} \pi_{d b}-N^{d} \pi_{d f} \gamma^{c f} \phi_{c a b} \\
& +2 m^{c d} m^{h f} \phi_{f d b} \phi_{h c a}-\gamma^{c d} \stackrel{\circ}{\nabla}_{c} \phi_{d a b} \\
& +2 \mathcal{T}_{a b}-2 \nabla_{(a} F_{b)},
\end{aligned}
$$

where it is understood that $N^{a}, \gamma^{a b}$ and $\gamma_{a}{ }^{b}$ have been substituted using equation (141) while $\mathcal{T}_{a b}$ and $\nabla_{(a} F_{b)}$ have been expanded out to order $O\left(h^{2}, h \stackrel{\circ}{\nabla} h,(\stackrel{\circ}{\nabla} h)^{2}\right)$. Observe that, in terms of the $T$ and $R$ coordinates one has $\stackrel{\circ}{N}_{a}=(\mathrm{d} T)_{a}$. Thus, for completeness, let $\stackrel{\circ}{R}_{a}=$ $(\mathrm{d} R)_{a}$. A direct computation shows that $\stackrel{\circ}{N}^{a} \phi_{a b c} \doteq 0$. The latter implies that one write

$$
\phi_{a b c} \doteq \stackrel{\circ}{R}_{a} \phi_{R b c}+S^{A}{ }_{a} \phi_{S_{A} b c},
$$

where $\phi_{R a b} \equiv \stackrel{\circ}{R}^{c} \phi_{c a b}$ and $\phi_{S_{A} a b} \equiv S_{A}^{c} \phi_{c a b}$. To derive the asymptotic system, following the strategy described in section IIIB, one defines

$$
\sigma_{a b}^{+}=\pi_{a b}+\phi_{R a b}, \quad \sigma_{a b}^{-}=\pi_{a b}-\phi_{R a b},
$$

and expresses the evolution equations 144 in terms of the variables

$$
\left\{\sigma_{a b}^{+}, \quad \sigma_{a b}^{-}, \quad \phi_{a b c}, \quad h_{a b}\right\} .
$$


Introducing the rescaled variables,

$$
\begin{aligned}
\Sigma_{a b}^{+} & =R^{2} \sigma_{a b}^{+}, & \Sigma_{a b}^{-} & =R \sigma_{a b}^{-}, \\
\Phi_{S_{A} a b} & =R^{2} \phi_{S_{A} a b}, & & H_{a b}=R h_{a b} .
\end{aligned}
$$

and defining

$$
\mathbb{T}_{a b}=R^{2} \mathcal{T}_{a b}
$$

one obtains, assuming as in section $\mathrm{V}$ that the gauge source functions decay sufficiently fast $F^{a} \sim R^{-3}$, the following expansions

$$
\begin{aligned}
& \stackrel{\circ}{\nabla}_{q} H_{a b}+\frac{1}{2} \Sigma_{a b}^{-} \simeq 0 \\
& -\frac{1}{2} H_{L L} \stackrel{\circ}{\nabla}_{q} \Sigma_{a b}^{-}+\gamma_{2}\left(H_{a b}+\frac{1}{2} \Sigma_{a b}^{+}-\stackrel{\circ}{\nabla}_{s} H_{a b}\right)+\stackrel{\circ}{\nabla}_{s} \Sigma_{a b}^{-} \\
& \simeq \frac{1}{4} \Sigma_{a L}^{-} \Sigma_{b L}^{-}-\frac{1}{2} \hat{L}_{(a} \Sigma_{b) h}^{-} \Sigma_{L}^{-h}+\frac{1}{8} \hat{L}_{a} \hat{L}_{b} \Sigma^{-c d} \Sigma_{c d}^{-}-2 \mathbb{T}_{a b}, \\
& \frac{1}{8}\left(H_{\underline{L L}}+H_{L L}-2 H_{L \underline{L}}\right) \stackrel{\circ}{\nabla}_{q} \Sigma_{a b}^{-}+\gamma_{2}\left(H_{a b}-\stackrel{\circ}{\nabla}_{s} H_{a b}+\right. \\
& \left.\frac{1}{2} \Sigma_{a b}^{+}\right)+2 \stackrel{\circ}{\nabla}_{q} \Sigma_{a b}^{+} \simeq \Sigma_{a b}^{-}+\frac{1}{16} \Sigma_{a b}^{-}\left(\Sigma_{L L}^{-}-3 \Sigma_{L L}^{-}-2 \Sigma_{L L}^{-}\right) \\
& -\frac{1}{4} \Sigma_{a L}^{-} \Sigma_{b L}^{-}+\frac{1}{2} L_{(a} \Sigma_{b) h}^{-} \Sigma_{L}^{-h}-\frac{1}{8} L_{a} L_{b} \Sigma^{-c d} \Sigma_{c d}^{-}+2 \mathbb{T}_{a b}, \\
& \stackrel{\circ}{\nabla}_{q} \Phi_{S_{A} a b}=-\frac{1}{2} \omega_{A}{ }^{c} \stackrel{\circ}{\nabla}_{c} \Sigma_{a b}^{-}-\gamma_{2}\left(\Phi_{S_{A} a b}-\omega_{A}{ }^{c} \stackrel{\circ}{\nabla}_{c} H_{a b}\right) .
\end{aligned}
$$

Now, to unwrap the connection of the asymptotic system in first order and second order form, define the rescaled reduction constraints as follows

$$
\mathbb{C}_{R a b}=R^{2} \mathcal{C}_{R a b}, \quad \mathbb{C}_{S_{A} a b}=R^{2} \mathcal{C}_{S_{A} a b},
$$

Then, a direct computation using equations (137), (144), (146) and (147), with the current decay assumptions about the gauge source functions $F_{a}$, one obtains

$$
\begin{aligned}
\Sigma_{a b}^{-} & \simeq-2 \stackrel{\circ}{\nabla}_{q} H_{a b}, \\
\Sigma_{a b}^{+} & \simeq-H_{a b}-\mathbb{C}_{R a b}+\stackrel{\circ}{\nabla}_{s} H_{a b} \\
& \quad+\frac{1}{8}\left(H_{\underline{L L}}-3 H_{L L}-2 H_{\underline{L} L}\right) \stackrel{\circ}{\nabla}_{q} H_{a b}, \\
\Phi_{S_{A} a b} & =-\mathbb{C}_{S_{A}}+\omega_{A}{ }^{c} \stackrel{\circ}{\nabla}_{c} H_{a b},
\end{aligned}
$$

where, written in full, these quantities satisfy,

$$
\Sigma_{a b}^{-}+\frac{\Sigma_{a b}^{+}}{R}=2 \stackrel{\circ}{\nabla}_{q} H_{a b}+\frac{2 \stackrel{\circ}{\nabla}_{s} H_{a b}}{R}-\frac{2 H_{a b}}{R}-\frac{2 \mathbb{C}_{R a b}}{R} .
$$

Substitution of the equations $(152)$ and 151 , into equation 149 and some rearranging reveals,

$$
\begin{aligned}
& \left(2 \stackrel{\circ}{\nabla}_{s}-H_{L L} \stackrel{\circ}{\nabla}_{q}\right) \stackrel{\circ}{\nabla}_{q} H_{a b} \simeq 2 \mathbb{T}_{a b}-\gamma_{2} \mathbb{C}_{R a b}-\stackrel{\circ}{\nabla}_{q} H_{a L} \stackrel{\circ}{\nabla}_{q} H_{b L} \\
& \quad+2 L_{(a} \stackrel{\circ}{\nabla}_{|q|} H_{b)}{ }^{c} \stackrel{\circ}{\nabla}_{q} H_{L c}-\frac{1}{2} L_{a} L_{b} \stackrel{\circ}{\nabla}_{q} H^{c d} \stackrel{\circ}{\nabla}_{q} H_{c d}, \\
& \quad \stackrel{\circ}{\nabla}_{q} H_{a b}+\frac{1}{2} \Sigma_{a b}^{-} \simeq 0, \\
& \stackrel{\circ}{\nabla}_{q} \mathbb{C}_{R a b} \simeq \gamma_{2} \mathbb{C}_{R a b}, \\
& \stackrel{\circ}{\nabla}_{q} \mathbb{C}_{S_{A} a b} \simeq \gamma_{2} \mathbb{C}_{S_{A} a b} .
\end{aligned}
$$

Setting $\gamma_{2} \sim R^{-1}$ and $\mathcal{T}_{a b}={ }^{\mathscr{I}} \mathcal{T}_{a b}$ as given in equation 80 and, following the philosophy of the asymptotic system by formally replacing the $\simeq$ by $=$ one obtains

$$
\begin{aligned}
& \left(2 \stackrel{\circ}{\nabla}_{s}-H_{L L} \stackrel{\circ}{\nabla}_{q}\right) \stackrel{\circ}{\nabla}_{q} H_{a b}=T_{a b}, \\
& \stackrel{\circ}{\nabla}_{q} H_{a b}+\frac{1}{2} \Sigma_{a b}^{-}=0, \\
& \stackrel{\circ}{\nabla}_{q} \mathbb{C}_{R a b}=0, \\
& \stackrel{\circ}{\nabla}_{q} \mathbb{C}_{S_{A} a b}=0,
\end{aligned}
$$

where $T_{a b}$ is the same tensor as that of equation 85 . Finally, from the last two equations in 154 one concludes that

$$
\mathbb{C}_{R a b}=\mathbb{C}_{R a b}^{\star}, \quad \mathbb{C}_{S_{A} a b}=\mathbb{C}_{S_{A} a b}^{\star},
$$

where $\mathbb{C}_{R}^{\star}=\left.\mathbb{C}_{R}\right|_{q=q_{\star}}, \mathbb{C}_{S_{A}}^{\star}=\left.\mathbb{C}_{S_{A}}\right|_{q=q_{\star}}$. Thus, assuming that the latter quantities are uniformly bounded, it follows from the analysis of subsection $\mathrm{VA}$ and expressions (151) and (147) that

$$
\begin{aligned}
\sigma_{\mathcal{U}}^{-} & \sim O\left(R^{-1-\omega}\right), & \sigma_{\mathcal{V}}^{-} & \sim O\left(R^{-1}\right), \\
\sigma_{\mathcal{U}}^{+} & \sim O\left(R^{-2}\right), & \sigma_{\mathcal{V}}^{+} & \sim O\left(R^{-2}\right), \\
\phi_{S_{A} \mathcal{U}} & \sim O\left(R^{-2}\right), & \phi_{S_{A} \mathcal{V}} & \sim O\left(R^{-2}\right), \\
h_{\mathcal{U}} & \sim O\left(R^{-1}\right) & h_{\mathcal{V}} & \sim O\left(R^{-1}\right) \\
\sigma_{\underline{L} \underline{L}}^{-} & \sim O\left(R^{-1} \ln R\right), & \sigma_{\underline{L L}}^{+} & \sim O\left(R^{-2} \ln R\right), \\
\phi_{S_{A} \underline{L L}} & \sim O\left(R^{-2} \ln R\right), & h_{\underline{L L}} & \sim O\left(R^{-1} \ln R\right),
\end{aligned}
$$

where $\mathcal{U} \in\left\{L L, L S_{A}, \varnothing\right\}, \mathcal{V} \in\left\{L \underline{L}, \underline{L} S_{A}, \times,+\right\}$.

For completeness notice that the generalized harmonic gauge condition (136) implies, to leading order, the following constraint equation

$$
m^{a b} \Gamma_{c a b}+F_{a} \doteq 0 .
$$

Assuming as before that $F_{a} \sim R^{-3}$ a direct calculation shows that the asymptotic form of these equations read

$$
\Sigma_{\mathcal{U}}^{-}=0
$$

Naturally, this implies that when the constraints are not violated we have $\sigma_{\mathcal{U}}^{-}=0$. Nonetheless, recall that, in the analysis of subsection $\mathrm{VA}$ does not rely on the satisfaction of the asymptotic GHG constraints. In other words, the analysis of subsection $\mathrm{VA}$ shows that even if small violations of the GHG constraints are present one has $\sigma_{\mathcal{U}}^{-}(\gamma) \sim O\left(R^{-1-\omega}\right)$ so that the coordinate lightspeed condition can be satisfied close to $\mathscr{I}$.

\section{THE TRAUTMAN-BONDI MASS}

In this section we discuss the implications of the present analysis for the definition of the Trautman-Bondi mass. The main issue to be analyzed here is if the $H_{\underline{L L}}$ component enters into the expression defining the Bondi mass and whether or not this affects its boundedness. 
Let $\mathcal{H}$ be an hyperboloidal slice in $\left(\mathcal{M}, g_{a b}\right)$ and let $\mathcal{S}^{2} \subset \mathcal{H}$ denote a surface of constant $R$ in $\mathcal{H}$. Recall the definitions introduced in section IV C for the $2+1+1$ split and define

$$
k \equiv q^{a b} \nabla_{a} R_{b} .
$$

where $q^{a b}=g^{a c} g^{b d} q_{c d}$. Additionally, let $\stackrel{\circ}{q}_{a b} \equiv \stackrel{\circ}{\gamma}_{a b}-\stackrel{\circ}{R}_{a} \stackrel{\circ}{R}_{b}$ and $\stackrel{\circ}{k} \equiv \stackrel{\circ}{q}^{a b} \nabla_{a} \stackrel{\circ}{R}_{b}$, where $\stackrel{\circ}{q}^{a b}=m^{a c} m^{b d} \stackrel{\circ}{q}_{c d}$, with $\stackrel{\circ}{\gamma}_{a b}$ and $\stackrel{\circ}{R}_{a}$ as defined in section VI. Using the above definitions, the Trautman-Bondi mass can be expressed as

$$
M=-\frac{1}{8 \pi} \int_{\mathcal{S}_{\infty}}(k-\stackrel{\circ}{k}) \mathrm{d} \mathcal{S}^{2}
$$

where d $\mathcal{S}^{2}$ denotes the area element in $\left(\mathcal{S}^{2}, q_{a b}\right)$ and $\mathcal{S}_{\infty}$ represents a cut of null-infinity [40. Consistent with the notation of subsection (IV C), some introducing arbitrary coordinates $\theta^{A}$ on $\mathcal{S}^{2}$, one has $\mathrm{d} \mathcal{S}^{2}=\sqrt{\operatorname{det}\left[q_{A B}\right]} \mathrm{d}^{2} \theta$. A direct computation using equation 76 gives

$$
\mathrm{d} S \simeq R^{2} \sqrt{\operatorname{det}\left[\sigma_{A B}\right]} \mathrm{d}^{2} \theta
$$

where $\sigma_{A B}$ denotes the standard metric on $\mathbb{S}^{2}$ in the $\theta^{A}$ coordinates. To have a more compact notation we denote the area element of $\mathbb{S}^{2}$ as $d \mathbb{S}^{2}$. Observe that, proceeding in analogous way as for the vector $N^{a}$ in section VI] one has the following expansions

$$
S_{a} \doteq \stackrel{\circ}{R}_{a}+\check{R}_{a}, \quad S^{a} \doteq \stackrel{\circ}{R}^{a}+\left(\check{R}^{a}-h^{a b} \stackrel{\circ}{R}_{b}\right)
$$

where,

$$
\check{R}_{a} \equiv\left(-\stackrel{\circ}{N}_{a} \stackrel{\circ}{N}^{b} \stackrel{\circ}{R}^{c}+\frac{1}{2} \stackrel{\circ}{R}_{a} \stackrel{\circ}{R}^{b} \stackrel{\circ}{R}^{c}\right) h_{b c} .
$$

Then, a direct computation shows that

$$
k-\stackrel{\circ}{k} \simeq \mathcal{K}+\frac{1}{2} C^{\underline{L}}-\frac{1}{2} \delta^{A B} \stackrel{\circ}{\nabla}_{a}\left(\frac{1}{R} H_{\underline{L}_{A}} \omega_{B}{ }^{a}\right),
$$

where

$\mathcal{K}=-\frac{H_{L L}}{4 R^{2}}-\frac{H_{\varnothing}}{4 R^{2}}+\frac{\partial_{s} H^{\varnothing}}{4 R^{2}}+\frac{1}{8 R^{2}}\left(2 \partial_{s}-H_{L L} \partial_{q}\right) H_{\underline{L L}}$.

and $C^{\underline{L}} \equiv \hat{L}_{a} C^{a}$ where $C^{a}$ encodes the GHG constraints (136). Observe that, the last term in (164) denotes the sum of the divergence of the vector fields $\frac{1}{R} H_{\underline{L} S_{A}} \omega_{B}{ }^{a}$ which can be regarded as vectors on $\mathcal{S}^{2}$. Consequently, the last term in (164) drops out after integration on $\mathcal{S}^{2}$.

Recall that in the calculation of the asymptotic constraint conditions 63 were obtained from the coefficient of the leading order term $R^{-1}$. Nonetheless, computed to second order $C \underline{L}$ reads

$$
\begin{aligned}
& C \underline{L} \simeq \frac{\partial_{q} H^{\varnothing}}{R}+\frac{1}{R^{2}}\left[H_{L \underline{L}}-\frac{1}{2} H_{\underline{L L}}+\frac{1}{2} H^{\varnothing}+H_{\underline{L} S_{A}} \stackrel{\circ}{\nabla}^{a} \omega^{A}{ }_{a}\right. \\
& +\delta^{A B} \omega_{A}{ }^{a} \stackrel{\circ}{\nabla}_{a} H_{\underline{L} S_{B}}+\frac{1}{2} H_{L \underline{L}} \partial_{q} H^{\varnothing}+\frac{1}{2} \partial_{s}\left(H^{\varnothing}-H_{\underline{L L}}\right) \\
& \left.-\partial_{q}\left(\left(H^{\times}\right)^{2}+\left(H^{+}\right)^{2}+\frac{1}{4}\left(H^{\varnothing}\right)^{2}-\frac{1}{4} H_{L L} H_{\underline{L L}}\right)\right]
\end{aligned}
$$

where it was assumed as usual that $F_{a} \sim R^{-3}$ so that the gauge source functions do not appear in the latter expansion. To simplify the subsequent discussion, from this point on-wards it will be assumed that the GHG constraints are satisfied to all orders so that $C^{a}=0$. Taking into account the latter observation one has

$$
M=-\frac{1}{8 \pi} \int_{\mathcal{S}_{\infty}} \mathcal{K} \mathrm{d} S
$$

To verify that the derived expression for $\mathcal{K}$ is correct observe that the mass loss formula can be recovered as follows. Using that $\partial_{T}=-\partial_{q}$ one obtains

$$
\partial_{T} M=\frac{1}{8 \pi} \int_{\mathcal{S}_{\infty}} \partial_{q} \mathcal{K} \mathrm{d} S
$$

Then, a direct computation using $C^{a}=0$ to replace $\partial_{q} H_{L L}$ and $\partial_{q} H^{\varnothing}$ and using equation (161), renders

$$
\partial_{T} M=\frac{1}{64 \pi} \int_{\mathbb{S}^{2}}\left(2 \partial_{s}-H_{L L} \partial_{q}\right) \partial_{q} H_{\underline{L L}} \mathrm{~d} \mathbb{S}^{2} .
$$

Using the asymptotic equation $87 \mathrm{c}$, one recovers the mass loss formula

$$
\partial_{T} M=-\frac{1}{16 \pi} \int_{\mathbb{S}^{2}}\left(\left(\partial_{q} H^{+}\right)^{2}+\left(\partial_{q} H^{\times}\right)^{2}\right) \mathrm{d} \mathbb{S}^{2} .
$$

As a side remark it is observed that the Hamiltonian and momentum constraints an be written in term of the GHG constraints and the asymptotic equations for $H_{\underline{L L}}$ and $H_{\underline{L} S_{A}}$.

Having verified that one can recover the mass loss formula, the main observation to be made is that $\mathcal{K}$ contains $H_{\underline{L L}}$ and, as discussed in subsection $\mathrm{VA}, H_{\underline{L L}} \sim$ $\ln R$. Nevertheless, the Trautman-Bondi mass is well defined. To see why this is the case, observe that, since $C^{a}=0$ and hence $\partial_{q} H_{L L}=0$, equation $87 \mathrm{c}$ can be written as

$$
\left(2 \partial_{s}-H_{L L} \partial_{q}\right) H_{\underline{L L}}=-4 \int_{q_{\star}}^{q}\left(\partial_{q} H^{+}\right)^{2}+\left(\partial_{q} H^{\times}\right)^{2} \mathrm{~d} \bar{q} .
$$

Substituting the latter expression into equation (165), one finds that $\mathcal{K}$ can be rewritten as

$$
\begin{aligned}
\mathcal{K}=\frac{1}{4 R^{2}}(- & \left.H_{L L}-H^{\varnothing}+\partial_{s} H^{\varnothing}\right) \\
& \quad-\frac{1}{2 R^{2}} \int_{q_{\star}}^{q}\left(\partial_{q} H^{+}\right)^{2}+\left(\partial_{q} H^{\times}\right)^{2} \mathrm{~d} \bar{q} .
\end{aligned}
$$

Thus, we observe that, despite that at first instance one would conclude that $M$ is diverging as it contains $H_{\underline{L L}}$, by virtue of the Einstein field equations, in this case, in the form of the asymptotic equation for $H_{\underline{L L}}$, the Trautman-Bondi mass is well defined.

Our final remark is that one can formally recover these results for the case in which there are small violations 
to the constraints $C^{a} \neq 0$ simply by redefining the Trautman-Bondi mass as

$$
M=-\frac{1}{8 \pi} \int_{\mathcal{S}_{\infty}}(k-\stackrel{\circ}{k})-\frac{1}{2} C \stackrel{L}{L} \mathrm{~d} \mathcal{S}^{2}
$$

and exploiting the results of subsection $\mathrm{VA}$, to use $\partial_{q} H_{\mathcal{U}} \sim O\left(R^{-\omega}\right)$ instead of $\partial_{q} H_{\mathcal{U}}=0$, in each of the previous computations.

\section{CONCLUSIONS}

The dual foliation formalism [22, 23, 41,43] is an approach to GR in which the tensor basis and choice of coordinates are left uncoupled. In [22] a proposal was given to use the formalism to help in the numerical treatment of future null-infinity via a suitably posed hyperboloidal initial value problem. Nevertheless, in order for this proposal to work, one of the requirements, found in [22], is that certain derivatives of the coordinate light-speed have enough decay. The latter condition is called the coordinate light-speed condition. Here we have studied whether or not one can expect the coordinate light-speed condition to be satisfied. This was done with the use of asymptotic expansions originally introduced in [29, 30] and employed in 24] to define the weak null condition. We have shown that the coordinate light-speed condition is related to the asymptotic harmonic constraints. As discussed in the main text, if the harmonic constraint condition is satisfied then the coordinate light-speed is trivially fulfilled. Nevertheless, numerical errors are inherent in free evolution schemes as one expects, albeit small, violations to the constraint equations. Moreover, constraint violations are expected to grow during the numerical evolution. Consequently we must analyze the system without assuming that the constraints are satisfied. It turns out that one cannot expect to satisfy the coordinate light-speed condition without modifying the field equations in such a way that one damps away constraint violations. Therefore we proposed a constraint addition such that resulting asymptotic system implies constraint damping in outgoing null directions. In other words we have shown that by adding specific multiples of the constraints, the asymptotic system predicts that the the coordinate light-speed condition will be satisfied. This paves the way for the explicit numerical treatment of future null-infinity. Although the constraint addition proposed was tailored for the purposes of the application in mind, it could be easily generalized and modified. In the original discussion of the weak null condition for the Einstein field equations in 24 one can classify the components of the metric perturbation $h_{a b}$ into "good" components and "bad" components. The good components are those whose equations satisfy the classical null condition of 44446] while the "bad" component satisfies an equation that fails to satisfy the null condition. A slower fall-off is hence expected this component. In our analysis we found that the price to pay to force damping of constraint violations close to $\mathscr{I}^{+}$, and subsequently fulfillment of the light-speed condition, is to add another layer to this structure. The components of $h_{a b}$ are now be classified across three categories, which we call "the good, the bad and the ugly". The equations for the components lying in the new "ugly" category are precisely those associated with the constraints.

Having established fall-off rates within the asymptotic system, we turned to the Trautman-Bondi mass, and recovered the mass loss formula. Although at first glance the Trautman-Bondi mass formula contains terms that could potentially blow up at $\mathscr{I}^{+}$, by virtue of the Einstein field equations it turns out to be well defined. Finally, in concordance with the outlook of the rest of the work, we discussed how to modify the definition of the Trautman-Bondi with constraints in such a way that one can reproduce the above remarks, even when small constraints violations are present.

\section{Acknowledgments}

We are grateful to Laura Bernard, Naresh Dadhich, Adrián del Río Vega, Guillaume Faye, Chris Moore, Shalabh, Juan Valiente Kroon and Alex Vañó-Viñuales for helpful discussions. DH also gratefully acknowledges support offered by IUCAA, Pune, where part of this work was completed. The work was partially supported by the FCT (Portugal) IF Program IF/00577/2015.
[1] R. Penrose, Phys. Rev. Lett. 10, 66 (1963).

[2] R. Penrose, in Relativity, Groups, and Topology (Les Houches, France, 1964), edited by C. DeWitt and B. DeWitt (Gordon and Breach, New York, 1964), pp. 565584.

[3] S. Husa, in The Conformal Structure of Spacetimes: Geometry, Analysis, Numerics, edited by J. Frauendiener and H. Friedrich (2002), vol. 604, chap. Problems and Successes in the Numerical Approach to the Conformal Field Equations.

[4] J. Stewart, Advanced general relativity (Cambridge Uni- versity Press, 1991).

[5] J. Frauendiener, Living Rev. Relativity 7 (2004).

[6] A. Ashtekar, ArXiv e-prints (2014), 1409.1800.

[7] J.-A. Valiente-Kroon, Conformal Methods in General Relativity (Cambridge University Press, Cambridge, 2016).

[8] A. Trautman, Bulletin of the Polish Academy of Sciences VI, 407 (1958).

[9] H. Bondi, M. G. J. van der Burg, and A. W. K. Metzner, Proc. Roy. Soc. A 269, 21 (1962).

[10] R. Sachs, Proc. Roy. Soc. London A270, 103 (1962). 
[11] B. P. Abbott et al., Phys. Rev. Lett. 116, 061102 (2016), 1602.03837

[12] J. Winicour, Living Rev. Relativity 15, 2 (2012), [Online article], URL http://www.livingreviews.org/ lrr-2012-2.

[13] H. Friedrich, Proc. Roy. Soc. London A 375, 169 (1981).

[14] G. Doulis and J. Frauendiener (2016), 1609.03584.

[15] A. Zenginoglu, Class. Quant. Grav. 25, 195025 (2008), 0808.0810

[16] A. Vañó-Viñuales, S. Husa, and D. Hilditch, Class. Quant. Grav. 32, 175010 (2015), 1412.3827.

[17] A. Vañó-Viñuales and S. Husa, J. Phys. Conf. Ser. 600, 012061 (2015), 1412.4801.

[18] A. Vañó-Viñuale, Ph.D. thesis, U. Iles Balears, Palma (2015), 1512.00776, URL http://inspirehep.net/ record/1407828/files/arXiv:1512.00776.pdf

[19] V. Moncrief and O. Rinne, Class.Quant.Grav. 26, 125010 (2009), 0811.4109.

[20] J. M. Bardeen, O. Sarbach, and L. T. Buchman, Phys. Rev. D83, 104045 (2011), 1101.5479.

[21] G. Calabrese, C. Gundlach, and D. Hilditch, Class.Quant.Grav. 23, 4829 (2006), gr-qc/0512149.

[22] D. Hilditch, E. Harms, M. Bugner, H. Rüter, and B. Brügmann, Class. Quant. Grav. 35, 055003 (2018), 1609.08949.

[23] D. Hilditch (2015), 1509.02071.

[24] H. Lindblad and I. Rodnianski, Comptes Rendus Mathematique 336, 901 (2003), ISSN 1631073X, URL http://www.sciencedirect.com/science/ article/pii/S1631073X03002310.

[25] J. Keir, ArXiv e-prints (2018), 1808.09982.

[26] O. Brodbeck, S. Frittelli, P. Hübner, and O. A. Reula, J. Math. Phys. 40, 909 (1999), gr-qc/9809023.

[27] C. Gundlach, J. M. Martin-Garcia, G. Calabrese, and I. Hinder, Class. Quantum Grav. 22, 3767 (2005), grqc/0504114.

[28] L. Lindblom, M. A. Scheel, L. E. Kidder, R. Owen, and O. Rinne, Class. Quant. Grav. 23, S447 (2006), grqc/0512093.

[29] L. Hörmander, The lifespan of classical solutions of nonlinear hyperbolic equations (Springer Berlin Heidelberg, Berlin, Heidelberg, 1987), pp. 214-280, ISBN 978-3-540-
47886-7, URL https://doi.org/10.1007/BFb0077745

[30] L. Hörmander, Lectures on Nonlinear Hyperbolic Differential Equations, Mathématiques et Applications (Springer Berlin Heidelberg, 1997), ISBN 9783540629214, URL https://books.google.pt/ books?id=qps $02 \mathrm{wnhmEMC}$

[31] C. Sogge, Lectures on nonlinear wave equations, no. Bd. 2 in Monographs in analysis (International Press, 1995).

[32] H. Lindblad and I. Rodnianski, Communications in Mathematical Physics 256, 43 (2005), math/0312479.

[33] H. Lindblad, Communications in Mathematical Physics 353, 135 (2017), URL https://doi.org/10.1007/ s00220-017-2876-z

[34] H. Lindblad and I. Rodnianski, ArXiv Mathematics eprints (2004), math/0411109.

[35] A. Weyhausen, S. Bernuzzi, and D. Hilditch, Phys. Rev. D 85, 024038 (2012), 1107.5539.

[36] http://www.tpi.uni-jena.de/ hild/AsHypDF.tgz.

[37] J. M. Martín-García, xAct: tensor computer algebra. (2017), http://www.xact.es/

[38] M. E. Taylor, Partial differential equations III: nonlinear equations (Springer Verlag, 1996).

[39] A. G.-P. Gómez-Lobo and J. A. Valiente Kroon, Journal of Geometry and Physics 58, 1186 (2008), 0712.3373.

[40] E. Poisson, A Relativist's Toolkit: The Mathematics of Black-Hole Mechanics (Cambridge University Press, 2004), ISBN 0521830915.

[41] D. Hilditch and R. Richter, Phys. Rev. D94, 044028 (2016), 1303.4783.

[42] D. Hilditch and M. Ruiz (2016), 1609.06925.

[43] A. Schoepe, D. Hilditch, and M. Bugner, Phys. Rev. D97, 123009 (2018), 1712.09837.

[44] S. Klainerman, Communications on Pure and Applied Mathematics 33, 43 (1980).

[45] S. Klainerman, in Nonlinear systems of partial differential equations in applied mathematics, Part 1 (Santa Fe, N.M., 1984) (Amer. Math. Soc., Providence, RI, 1986), vol. 23 of Lectures in Appl. Math., pp. 293-326.

[46] D. Christoudolou, Communications in Mathematical Physics 105, 337 (1986). 